\title{
A Standard of Small Capacitance
}

\author{
By Chester Snow
}

\begin{abstract}
A formula is derived for computing the electrical capacitance of an absolute standard that is a modification of the ordinary parallel-plate condenser with coplanar guard. The modification consists in placing the circular face of the electrode at the bottom of a cylindrical hole in the guard. The analogous two-dimensional arrangement is also considered. The two cases are formulated as special cases of a more general one, in such a manner as to show that certain approximate formulas have errors of the same order of magnitude in each case. The magnitude of the error is then determined by comparison with an exact formula obtained for the two-dimensional case by conformal transformation. With certain restrictions, easily made in practice, this error seems to be less than one part in two thousand.
\end{abstract}

\section{Introduction}

To meet the demand for a standard of very small electrical capacitance that may be computed from its dimensions, the ordinary parallel-plate type with coplanar guard has been modified by placing one electrode at the bottom of a cylindrical hole (or well) in the guard as shown in figure 1, a. A formula is required for computing the coefficient of capacitance $C$ between the conductor on the left and the rod that snugly fits the cylindrical hole.

For the mathematical formulation of the problem it has been idealized in two ways. Further restrictions are made, but these are easy to meet in practice.

If the radius of the disk on the left in figure 1, a, is sufficiently large compared to its distance from the guard, there will be a region between them where the electrostatic field is nearly uniform. If it were perfectly uniform, the distribution of charge at the edge of the hole, in the cavity, and on the piston would be the same as if the radius of the left-hand conductor and guard were infinite. This is the first simplification in passing from figure $1, \mathrm{a}$, to the diagram in figure $1, \mathrm{~b}$. In the latter it is sufficient to show only the half of a meridian section, since the potential has axial symmetry. The most sensitive experimental tests show that when the radius in figure 1, a (corresponding to $A_{2} A_{0}$ in fig. $1, \mathrm{~b}$ ) is five times the

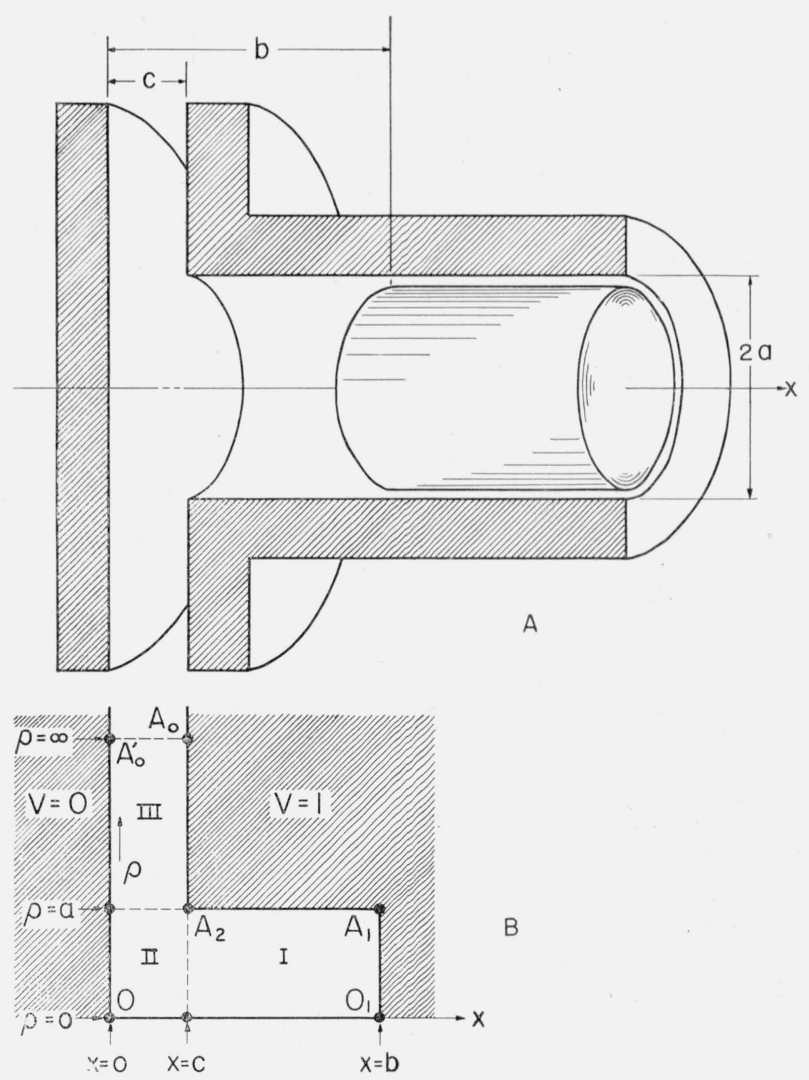

FIGURE 1. The dielectric volume is generated by rotation around the $x$-axis of the plane region $I+I I+I I I$.

A, Isometric drawing of capacitor; B, meridian section in the half-plane of the cylindrical coordinates $(X, \rho)$. 
separation, $c$, there is no detectable change in capacitance by further increase in this ratio.

The second assumption, as shown in figure $1, b$, is that the clearance between the piston and its guard may be ignored. The two are kept at the same potential but insulated from each other. Condensers of this type have been constructed in which this insulation is several hundred megohms, although the clearance is almost invisible to the naked eye. If the hole were very shallow, the edge $A_{1}$ being close to $A_{2}$, a correction for clearance would be more important, but if the depth of the hole were of the order of magnitude of its radius, both being large compared to the clearance, such a correction appears to be unimportant. With no clearance, the electric field vanishes at the edge $A_{1}$. There is another reason for making the hole relatively deep, say greater than its radius or one-half of its radius. This arises out of purely mathematical difficulties.

After adopting these two simplifications and formulating the potential, on the basis indicated in figure $1, \mathrm{~b}$, a formula for the capacitance $C$ was obtained in terms of known functions and an infinite set of positive constants $x_{s}(s=1,2,3$. . . $\infty)$, which are the solutions of an infinite system of linear equations. The formulation as an integral equation is given in the appendix. It suggested the proof, which is given, that the solution by the method of successive substitutions would be convergent for all positive real values of the depth-ratio, $\beta=(b-c) / a$, and of the spacingratio, $\gamma=c / a$. The computation of the iterated series, which give the constants $x_{s}$, seems to be very laborious in general. To obtain a simple, explicit formula for each $x_{s}$ (and therefore for capacitance $C$ ) it was assumed that $0 \leqslant \gamma \leqslant 1 / 5$ and $1 \leqslant \beta \leqslant \infty$, or roughly of this order. The shallow hole is again excluded, and the restriction on $\gamma$ is such as must be made in practice even for the coplanar case.

These restrictions do not prevent the extension of the range of standard capacitance down to the lowest desirable values. Since there are three adjustable lengths $a, b$, and $c$, it is also practicable to make a capacitor to which our formula applies, whose value $C$ is as large as that of a coplanar type with smaller radius $a$. Each capacitance could be computed and they could be compared experimentally, so there is a possibility of experimental check on the restricted formula for $C$ that is given here. (We have no practicable formula of precision for the case of a relatively shallow hole.)

It is not desirable to construct a set of standards of very low values, whose capacitance must be found experimentally by stepping down from the parallel-plate type. Hence the estimate of the order of magnitude of error in the restricted formula for $C$ (eq 35) should be based on mathematical considerations if possible. Such an estimate is made here by what amounts to a mathematical experiment.

To make this we consider the corresponding two-dimensional arrangement, which may also be represented by the half-plane of figure $1, \mathrm{~b}$, viewing it as a cross section of endless conductors, $x$ and $\rho$ now being rectangular coordinates instead of the cylindrical coordinates used before when the $x$ axis was the axis of symmetry. On this figure the two-dimensional potential has the same boundary values as before, but instead of the axially symmetric potential equation

$$
\left(D_{x}^{2}+D_{x}^{2}+\frac{1}{\rho} D_{\rho}\right) V=0
$$

we now have

$$
\left(D_{x}^{2}+D_{\rho}^{2}\right) \quad V=0 .
$$

The formulation of this problem in every step is analogous to that used before. A parallel treatment of the two cases, which was first used, was later abandoned in favor of the combined treatment given here, the two potential equations now being special cases of a more general one. The analogue of $C / a$ is formally $\pi C^{\prime}$, where $C^{\prime}$ is the capacitance per unit length of slot (the analogue of the cylindrical hole of diameter $2 a$, being the endless slot of width $2 a$ ). The general formula gives $C / a$ (eq 25 below) as the same function of $\beta$ and $\gamma$ and the roots $\alpha_{s}$ of $J_{0}(\alpha)=0$, that $\pi C^{\prime}$ is of $\beta$ and $\gamma$ and the roots $\alpha_{s}^{\prime}$ of $\cos \alpha=0$. The same is true of the restricted formulas 35 and $35 \mathrm{a}$. The combined formulation of the two cases makes it evident that the error is of the same order of magnitude in both cases for the restricted formulas 35 and 35 a.

But it is known that the two-dimensional problem may be solved by conformal transformation with integrals of elliptic functions, and this leads to a formula by which $\pi C^{\prime}$ may be computed with any desired precision. From this it appears that 
the restricted formula $35 \mathrm{a}$ (and hence eq 35) is in error by not more than one part in two thousand (when $0<\gamma \leqslant \frac{1}{5}$ and $1<\beta<\infty$ ).

Capacitors that approximate to each of the two types here considered have been constructed by C. Moon, and their coefficients $C$ and $C^{\prime}$ measured by comparison with the parallel-plate type. The values agree with those computed by the restricted formulas given here, the experimental error being estimated as one part in a thousand. The longish, two-dimensional type is more difficult to construct with precision.

\section{Combined Formulation of the Two Potential Problems}

The potential $V(x, \rho)$ must satisfy the boundary conditions

$$
\begin{array}{ll}
V(o, \rho)=0 & \text { for } o \leqslant \rho \leqslant \infty \\
V(b, \rho)=1 & \text { for } o \leqslant \rho \leqslant a \\
V(x, a)=1 & \text { for } c \leqslant x \leqslant b \\
V(c, \rho)=1 & \text { for } a \leqslant \rho \leqslant \infty
\end{array}
$$

$D_{\rho} V=0$ when $\rho=0$, and $V \rightarrow x / c$ when $\rho \rightarrow \infty$.

In the dielectric region the axially symmetric potential $V$ satisfies the partial differential equation

$$
\left(D_{x}^{2}+D^{2}\right) V+\frac{1}{\rho} D_{\rho} V=0 .
$$

The two-dimensional potential has the same boundary conditions but satisfies the equation

$$
\left(D_{x}^{2}+D_{\rho}^{2}\right) V=0 .
$$

They may be considered as special cases, $\nu=0$ and $\nu=-1 / 2$ of $V(x, \rho)$, which has the same boundary conditions and is a solution of

$$
\left(D_{x}^{2}+D_{\rho}^{2}\right) V+\frac{1+2 \nu}{\rho} D_{\rho} V=0 .
$$

As far as the formulation of the problem is concerned, it is only necessary to assume that the constant parameter $\nu$ is real and greater than -1 .

With this restriction let $\alpha_{s}$ denote the $s^{\text {th }}$ positive root of $J_{\nu}(\alpha)=0$. It is known that for the interval $0<\xi<1$, the set of normal functions (with weighting factor $\xi$ )

$$
\phi_{s}(\xi) \equiv \sqrt{2} \frac{J_{\nu}\left(\alpha_{s} \xi\right)}{J_{\nu+1}\left(\alpha_{s}\right)}(s=1,2,3, \ldots \infty),
$$

form a closed set, and they are normalized, for

$$
\int_{0}^{\prime} \xi \phi_{s}(\xi) \phi_{n}(\xi) d \xi=\delta_{n s}=1 \text { if } n=s,=0 \text { if } n \neq s .
$$

Another independent closed set, associated with the same constants is

$$
\psi_{s}(\xi) \equiv \sqrt{2} \frac{J_{v+1}\left(\alpha_{s} \xi\right)}{J_{v+1}\left(\alpha_{s}\right)} .
$$

We use $J_{\nu}(t)$ and $H_{\nu}(t)$ to denote Bessel's cylinder function of $t$ and the first kind of Hankel's function, and assume that the $t$-plane is cut along its negative real axis. Between these two functions and their derivatives, there is the identical relation in $t$,

$$
\frac{J_{\nu}^{\prime}(t)}{J_{\nu}(t)}-\frac{H_{\nu}^{\prime}(t)}{H_{\nu}(t)}=\frac{2}{i \pi t H_{\nu}(t) J_{\nu}(t)} .
$$

There is also the identity in $t$,

$$
2 \sum_{s=1}^{\infty} \frac{J_{\nu+n}\left(\alpha_{s} \xi\right)}{\alpha_{s}{ }^{n-1}\left(\alpha_{s}^{2}-t^{2}\right) J_{\nu+1}\left(\alpha_{s}\right)}=\frac{J_{\nu+n}(t \xi)}{t^{n} J_{\nu}(t)},
$$

which is valid for $0 \leqslant \xi \leqslant 1$, if $n=1,2,3, \ldots$ and for $0 \leqslant \xi<1$ in case $n=0$, which becomes

$$
2 \sum_{s=1}^{\infty} \frac{\alpha_{s} J_{\nu}\left(\alpha_{s} \xi\right)}{\left(\alpha_{s}^{2}-t^{2}\right) J_{\nu+1}\left(\alpha_{s}\right)}=\frac{J_{\nu}(t \xi)}{J_{\nu}(t)} \text { for } 0 \leqslant \xi<1 .
$$

Letting $t \rightarrow 0$ in this gives

$$
2 \sum_{s=1}^{\infty} \frac{J_{\nu}\left(\alpha_{s} \xi\right)}{\alpha_{s} J_{\nu+1}\left(\alpha_{s}\right)}=\xi^{\nu} \text { for } 0 \leqslant \xi<1 .
$$

For brevity let $\xi=\rho / a$

$$
\beta \equiv \frac{b-c}{a} \text { and } \gamma \equiv \frac{c}{a} .
$$

In the appendix is a formulation by contour integrals leading to an integral equation. The much shorter discussion given here contains all that is essential without reference to the appendix.

Consider the particular solutions $V_{s}(x, \rho) \quad(s=1$, $2,3 \ldots$. .), which vanish on all the boundaries of the dielectric and are continuous throughout, but have discontinuities in $\partial V / \partial x$ at the plane $x=c$, corresponding to a surface density $\sigma_{s}$ at this plane, where

$$
4 \pi \sigma_{s}=\left(\frac{\partial V}{\partial x}\right)_{x=e-0}-\left(\frac{\partial V}{\partial x}\right)_{x=e+0} .
$$


Assume in region $I$ it is

$$
V_{s}(x, \rho)=\frac{\sinh \alpha_{s}(b-x) / a}{\alpha_{s} \sinh \beta \alpha_{s}} \cdot \frac{\xi^{-\nu} J_{\nu}\left(\alpha_{s} \xi\right)}{J_{\nu+1}\left(\alpha_{s}\right)} ;
$$

then in region II it must be

$V_{s}(x, \rho)=\frac{\sinh \alpha_{s} x / a}{\alpha_{s} \sinh \gamma \alpha_{s}} \cdot \frac{\xi^{-\nu} J_{\nu}\left(\alpha_{s} \xi\right)}{J_{\nu+1}\left(\alpha_{s}\right)}+\pi \gamma \xi^{-\nu}$.

$\sum_{n=1}^{\infty}(-1)^{n+1} \sin \left(\frac{n \pi x}{c}\right) \frac{(i n \pi / \gamma) H_{\nu}(i n \pi / \gamma) J_{\nu}(i n \pi \xi / \gamma)}{\left[(n \pi)^{2}+\left(\gamma \alpha_{s}\right)^{2}\right]}$

and in region III it must be

$V_{s}(x, \rho)=\pi \gamma \xi^{-\nu}$

$\sum_{n=1}^{\infty}(-1)^{n+1} \sin \left(\frac{n \pi x}{c}\right) \frac{(i n \pi / \gamma) H_{\nu}(i n \pi \xi / \gamma) J_{v}(i n \pi / \gamma)}{\left[(n \pi)^{2}+\left(\gamma \alpha_{s}\right)^{2}\right]}$

By use of the identities (1) and (2a), each for $t=(i n \pi / \gamma)$, it may be verified that not only $V_{s}$ but also $\partial V_{s} / \partial \rho$ is continuous at $\rho=a$. Also, since $V_{s}$ is continuous at $x=c$, it is found that the surface density $\sigma_{s}$ is given by

$$
\begin{gathered}
4 \pi a \xi^{\nu} \sigma_{s}=\left(\operatorname{coth} \gamma \alpha_{s}+\operatorname{coth} \beta \alpha_{s}\right) \frac{J_{\nu}\left(\alpha_{s} \xi\right)}{J_{\nu+1}\left(\alpha_{s}\right)}- \\
\gamma^{2} \sum_{k=1}^{\infty} \alpha_{k} X_{s k}^{(1)} \frac{J_{\nu}\left(\alpha_{k} \xi\right)}{J_{\nu+1}\left(\alpha_{k}\right)}
\end{gathered}
$$

where the positive real coefficients are functions of $\gamma$ defined by:

$$
X_{s k}^{(1)}=X_{k s}^{(1)}=\sum_{n=1}^{\infty} \frac{2 n \pi^{2}(i n \pi / \gamma) H_{\nu}(i n \pi / \gamma) J_{\nu}\left(i n \pi^{\prime} \gamma\right)}{\left[(n \pi)^{2}+\left(\gamma \alpha_{s}\right)^{2}\right] \cdot\left[(n \pi)^{2}+\left(\gamma \alpha_{k}\right)^{2}\right]} .
$$

The required potential $V_{\nu}$ may be represented in the form

$$
\begin{aligned}
V_{\nu}(x, \rho)= & \frac{x}{c}-2 \sum_{s=1}^{\infty} x_{s} V_{s}(x, \rho) \text { in II and III }= \\
& 1-2 \sum_{s=1}^{\infty} x_{s} V_{s}(x, \rho) \text { in } \mathrm{I}
\end{aligned}
$$

where the real constants $x_{s}$ must be so chosen that $\partial V_{\nu} / \partial x$ is continuous at $x=c$, that is

$$
2 \sum_{s=1}^{\infty} x_{s}\left(4 \pi a \sigma_{s}\right)=\frac{1}{\gamma} \text { for } 0 \leqslant \xi<1,
$$

or by eq 5

$$
\begin{aligned}
& 2 \sum_{s=1}^{\infty} \frac{J_{\nu}\left(\alpha_{s} \xi\right)}{J_{\nu+1}\left(\alpha_{s}\right)} \cdot \cdot \\
& {\left[\left(\operatorname{coth} \gamma \alpha_{s}+\operatorname{coth} \beta \alpha_{s}\right) x_{s}-\gamma^{2} \alpha_{s} \sum_{k=1}^{\infty} X_{s k}^{(1)} \cdot x_{k}\right]=}
\end{aligned}
$$

$$
\frac{\xi^{\nu}}{\gamma}=(2 / \gamma) \sum_{1}^{\infty} J_{\nu}\left(\alpha_{s} \xi\right) /\left[\alpha_{s} J_{\nu+1}\left(\alpha_{s}\right)\right] . \text { by }
$$

If, for brevity, we place

$$
A_{s} \equiv \frac{1}{\operatorname{coth} \gamma \alpha_{s}+\operatorname{coth} \beta \alpha_{s}}=\frac{\sinh \beta \alpha_{s} \sinh \gamma \alpha_{s}}{\sinh (\beta+\gamma) \alpha_{s}},
$$

the system of linear equations that determine the $x_{s}$ may be written

$x_{s}=A_{s}\left[\frac{1}{\gamma \alpha_{s}}+\gamma^{2} \alpha_{s} \sum_{k=1}^{\infty} X_{s k}^{(1)} x_{k}\right]$ for $s=1,2,3, \ldots \infty$.

By "successive substitutions" this system is found to be equivalent to

$$
\begin{aligned}
x_{s}= & \frac{\alpha_{s} A_{s}}{\gamma}\left[\frac{1}{\alpha_{s}{ }^{2}}+\gamma^{2} \sum_{k=1}^{\infty} \frac{X_{s k}^{(1)} A_{k}}{\alpha_{k}}+\gamma^{4} \sum_{k=1}^{\infty} \frac{X_{s k}^{(2)} A_{k}}{\alpha_{k}}+\ldots\right. \\
& \left.\gamma^{2(m-1)} \sum_{k=1}^{\infty} \frac{X_{\mathrm{sk}}^{(m-1)} A_{k}}{\alpha_{k}}+\gamma^{2(m-1)} \sum_{k=1}^{\infty} X_{s k}^{(m)} x_{k}\right],
\end{aligned}
$$

where $m$ is any positive integer; in case $m=1$ this reduces to eq 9 .

The iterated coefficients are positive reals, defined by

$$
X_{s k}^{(m+1)}=X_{k s}^{(m+1)}=\sum_{r=1}^{\infty} \alpha_{r} A_{r} X_{r s}^{(1)} X_{r k}^{(m)},
$$

where $X_{s / o}^{(1)}$ is defined in eq 6 .

If $m \rightarrow \infty$, the second number of eq 10 becomes an infinitive series that either converges or diverges to $+\infty$ (it cannot oscillate since all the terms are positive). If the series converges it gives the solution of the system (eq 9).

To examine this question let $x_{s}^{(1)}, x_{s}^{(2)}, \ldots . x_{s}^{(m)}$ $(s=1,2,3 \ldots \infty)$ each denote an infinite set of positive constants, each being derived from its predecessor by the operation that is the second member of eq 9 .

$$
x_{s}^{(m+1)}=\frac{\alpha_{s} A_{s}}{\gamma}\left[\frac{1}{\alpha_{s}^{2}}+\gamma^{3} \sum_{k=1}^{\infty} X_{s k}^{(1)} x_{k}^{(m)}\right] .
$$

Starting from any bounded initial set $x_{\mathrm{s}}^{(1)}$, otherwise arbitrary, say

$$
0<x_{s}^{(1)} \leqslant M^{(1)},
$$

the repeated application of this operation gives

$$
x_{s}^{(m+1)}=\frac{\alpha_{s} A_{s}}{\gamma}\left[\frac{1}{\alpha_{s}^{2}}+\gamma^{2} \sum_{k=1}^{\infty} \frac{X_{s k}^{(1)} A_{k}}{\alpha_{k}}+\gamma^{4} \sum_{k=1}^{\infty} \frac{X_{s k}^{(2)} A_{k}}{\alpha_{k}}+\ldots+\right.
$$




$$
\left.\gamma^{2(m-1)} \sum_{k=1} \frac{X_{s k}^{(m-1)} A_{k}}{\alpha_{k}}+\gamma^{2 m+1} \sum_{k=1}^{\infty} X_{s k}^{(m)} x_{k}^{(1)}\right]
$$

If we take the initial set, $x_{s}^{(1)} \equiv 1$, this becomes the second member of eq. 10, but it is here assumed merely that $x_{s}^{(1)}$ is bounded.

If

$$
0<x_{s}^{(m)} \leqslant M^{(m)} \text { for every } s,
$$

we may then prove that

$0<x_{s}^{(m+1)}<M^{(m)}-1$ for every $s$, when $M^{(m)}>2(13 \mathrm{a})$

and

$0<x_{s}^{(m+1)}<1$ for every $s$, when $M^{(m)} \leqslant 2$.

This proposition will be shown to hold for every finite value of the positive constants $\beta$ and $\gamma$, but in the proof we exclude the case $\beta=\gamma=0$ in which there is no problem, and also the limiting case, $\gamma \rightarrow 0,0<\beta<\infty$, in which the solution of eq 10 is obviously $x_{s}=1$ for every $s$.

Before attempting a detailed proof, we may notice that if eq 13a is true, it then follows that, whatever the initial set (provided it is bounded), we shall arrive at an integer $m$ for which $M^{(m)} \leqslant 2$. Proceeding to larger values of $m$ we find that if eq $13 \mathrm{~b}$ is true then an integer $m$ exists such that for every larger integer $m$ the set $x_{s}^{(m+1)}$ remains bounded and less than 1 . Combining this with the fact that $x_{s}^{(m+1)}$ continually increases with increasing $m$, it is evident that the set $x_{s}^{(m+1)}$ approaches a limit and the second members of eq $12 \mathrm{~b}$ and 10 become a convergent infinite series of positive terms that is the solution of the set of eq 9 irrespective of the arbitrary initial set with which we start. In fact, certain initial sets $x_{\mathrm{s}}{ }^{(1)}$ may be found, which make say $x_{s}^{(2)}$ or $x_{s}^{(3)}$ a closer approximation to the solution than would be obtained by starting with the initial set suggested by eq 10 , namely,

$$
x_{s}^{(1)}=\frac{A_{s}}{\gamma \alpha_{s}} .
$$

It would follow that this solution must lie in the interval

$$
\frac{A_{s}}{\gamma \alpha_{s}}<x_{s}<1 \text { for every } s \text {. }
$$

This inequality shows that the remainder for a given $m$, (the last series in eq 10), has an upper bound

$$
\gamma^{2 m+1} \sum_{k=1}^{\infty} X_{s k}^{(\mathrm{m})} x_{k}<\gamma^{2 m+1} \sum_{k=1}^{\infty} X_{s / k}^{(m)}
$$

From eq 8 and 11 it is evident that all tbe positive quantities $X_{s k}^{(m)}$ are functions of $\beta$ and $\gamma$, except that $X_{s k}^{(1)}$ by its definition (eq 6) depends on $\gamma$ only.

To prove the propositions of eq $13 \mathrm{a}$ and $13 \mathrm{~b}$ we start with the inequality

$$
\begin{aligned}
& X_{s}^{(m+1)}<\frac{\alpha_{s} A_{s}}{\gamma}\left[\frac{1}{\alpha_{s}^{2}}+\gamma^{3} M^{(m)} \sum_{k=1}^{\infty} X_{s k}^{(1)}\right]= \\
& \frac{A_{s}}{\gamma \alpha_{s}}+\alpha_{s} A_{s} M^{(m)} \sum_{n=1}^{\infty} \\
& \frac{n \pi\left[\pi\left(\frac{i n \pi}{\gamma}\right) H_{\nu}\left(\frac{i n \pi}{\gamma}\right) J_{\nu}\left(\frac{i n \pi}{\gamma}\right)\right]}{(n \pi)^{2}+\left(\gamma \alpha_{s}\right)^{2}} \sum_{k=1}^{\infty} \frac{2}{\alpha_{k}^{2}+(n \pi / \gamma)^{2}}
\end{aligned}
$$

If, in the identity (eq 2), we take $n=1, \xi=1$, and $t=i n \pi / \gamma$, it becomes

$$
\sum_{k=1}^{\infty} \frac{2}{\alpha_{k}^{2}+\left(\frac{n \pi}{\gamma}\right)^{2}}=\frac{J_{\nu+1}(i n \pi / \gamma)}{(i n \pi / \gamma) J_{\nu}(i n \pi) \gamma} .
$$

Hence, for every $s$,

$$
\begin{aligned}
& x_{s}^{(m+1)}<\frac{\alpha_{s} A_{s}}{\gamma} . \\
& {\left[\frac{1}{\alpha_{s}^{2}}+M^{(m)} \gamma^{2} \sum_{n=1}^{\infty} \frac{\pi(n \pi / \gamma) H_{\nu}(i n \pi / \gamma) J_{\nu+1}(i n \pi / \gamma)}{(n \pi)^{2}+\left(\gamma \alpha_{s}\right)^{2}}\right] .}
\end{aligned}
$$

It will appear presently that

$$
0<\pi\left(\frac{n \pi}{\gamma}\right) H_{\nu}\left(\frac{i n \pi}{\gamma}\right) J_{\nu+1}\left(i n \frac{\pi}{\gamma}\right)<1
$$

for every finite positive integer $n$, this positive real quantity being a monotone increasing function of $\frac{n \pi}{\gamma}$, which only reaches 1 in the limit $n \rightarrow \infty$.

Hence

$$
x_{s}^{(m+1)}<\frac{\alpha_{s} A_{s}}{\gamma}\left[\frac{1}{\alpha_{s}^{2}}+\frac{M^{(m)}}{2 \alpha_{s}^{2}} \sum_{n=1}^{\infty} \frac{2\left(\gamma \alpha_{s}\right)^{2}}{(n \pi)^{2}+\left(\gamma \alpha_{s}\right)^{2}}\right]
$$

or since

$$
\sum_{n=1}^{\infty} \frac{2\left(\gamma \alpha_{s}\right)^{2}}{(n \pi)^{2}+\left(\gamma \alpha_{s}\right)^{2}}=\gamma \alpha_{s} \operatorname{coth} \gamma \alpha_{s}-1
$$


and $A_{s}=1 /\left(\operatorname{coth} \gamma \alpha_{s}+\operatorname{coth} \beta \alpha_{s}\right)$ by eq 8 , this becomes

$$
0<x_{s}^{(m+1)}<\frac{1+\frac{M^{(m)}}{2}\left(\gamma \alpha_{s} \operatorname{coth} \gamma \alpha_{s}-1\right)}{\gamma \alpha_{s}\left(\operatorname{coth} \gamma \alpha_{s}+\operatorname{coth} \beta \alpha_{s}\right)}
$$

for $s=1,2,3, \ldots \infty$.

From this, the second part of the theorem to be proved, eq $13 \mathrm{~b}$, follows directly, for if $M^{(m)} \leqslant 2$, this becomes

$$
\begin{aligned}
\frac{A_{s}}{\gamma \alpha_{s}}=\frac{1}{\gamma \alpha_{s .}\left(\operatorname{coth} \gamma \alpha_{s}+\operatorname{coth} \beta \alpha_{s}\right)} & <x_{s}^{(m+1)}< \\
& \frac{\operatorname{coth} \gamma \alpha_{s}}{\operatorname{coth} \gamma \alpha_{s}+\operatorname{coth} \beta \alpha_{s}}
\end{aligned}
$$

for every $s$.

To prove eq 13a, the four expressions

$\frac{M^{(m)}}{2}-1, M^{(m)}-2, \gamma \alpha_{s} \operatorname{coth} \gamma \alpha_{s}$ and $\gamma \alpha_{s} \operatorname{coth} \gamma \alpha_{s}-1$

are each positive when $M^{(m)}>2$.

Hence

$\left(M^{(m)}-1\right) \gamma \alpha \operatorname{coth} \beta \alpha+\left(\frac{M^{(m)}}{2}-1\right)(\gamma \alpha \operatorname{coth} \gamma \alpha-1)+$

$$
M^{(m)}-2>0 .
$$

Adding $\frac{M^{(m)}}{2}(\gamma \alpha \operatorname{coth} \gamma \alpha-1)$ to both sides gives

$$
\begin{aligned}
& 1+\frac{M^{(m)}}{2}\left(\gamma \alpha_{s} \operatorname{coth} \gamma \alpha_{s}-1\right) \\
& <\left(M^{(m)}-1\right) \gamma \alpha_{s}\left(\operatorname{coth} \gamma \alpha_{s}+\operatorname{coth} \beta \alpha_{s}\right) .
\end{aligned}
$$

The inequality (eq 16) then becomes eq 13a, which was to be proved.

Consequently the second member of eq 10 when $m \rightarrow \infty$ gives the solution $x_{s}$ of eq 9 as a convergent infinite series of positive terms, which may be written

$x_{s}=\frac{A_{s}}{\gamma \alpha_{s}}\left\{1+\left(\gamma \alpha_{s}\right)^{2} \sum_{k=1}^{\infty} \frac{A_{k}}{\alpha_{k}}\left[X_{s k}^{(1)}+\gamma^{2} X_{s k}^{(2)}+\gamma^{4} X_{s k}^{(3)}+\ldots\right]\right\}$.

where $A_{s}, X_{s k}^{(1)}$ and $X_{s k}^{(m)}$ may be computed by eq 8,6 , and 11 . The process is straightforward, the series converges for every positive $\beta$ and $\gamma$ and is therefore the complete solution of the general problem, but as such is practically useless in all its generality. By good luck we may restrict the problem so that only the first series of eq 17 is necessary.
Eq 2 with $n=1, \xi=1$ and $t=i n \pi / \gamma$, becomes

$$
2\left(\frac{i n \pi}{\gamma}\right) J_{\nu}\left(\frac{i n \pi}{\gamma}\right) \sum_{k=1}^{\infty} \frac{1}{\alpha_{k}^{2}+\left(\frac{n \pi}{\gamma}\right)^{2}}=J_{\nu+1}\left(\frac{i n \pi}{\gamma}\right) .
$$

Using this, the definition eq 6 of $X_{s k}^{(1)}$ leads to the exact equation,

$$
\gamma \sum_{k=1}^{\infty} X_{s k}^{(1)}=\sum_{n=1}^{\infty} \frac{\pi(n \pi / \gamma) H_{\nu}(i n \pi / \gamma) J_{\nu+1}(i n \pi / \gamma)}{(n \pi)^{2}+\left(\gamma \alpha_{s}\right)^{2}} .
$$

To complete the proof it is necessary to show that the inequality eq 15 is satisfied in both cases, $\nu=0$, and $\nu=-1 / 2$.

In the case $\nu=0, J_{0}\left(\alpha_{s}\right)=0$, the two positive real quantities have the asymptotic expansions

$$
\begin{array}{r}
\pi\left(\frac{i n \pi}{\gamma}\right) H_{0}\left(\frac{i n \pi}{\gamma}\right) J_{0}\left(\frac{i n \pi}{\gamma}\right) \sim 1+\frac{1}{8}\left(\frac{\gamma}{n \pi}\right)^{2} \\
\pi\left(\frac{n \pi}{\gamma}\right) H_{0}\left(\frac{i n \pi}{\gamma}\right) J_{1}\left(\frac{i n \pi}{\gamma}\right) \sim 1-\frac{1}{2}\left(\frac{\gamma}{n \pi}\right)-\frac{3}{16}\left(\frac{\gamma}{n \pi}\right)^{3} .
\end{array}
$$

If $\gamma \gtrless 1 / 5$ these are in error (in the most unfavorable case $n=1$ ) by less than one part in $10^{5}$.

From tables of $\mathrm{H}_{0}(i x)$ and $J_{1}(i x)$ for $x>0$, it may be verified that the first member of eq 19a is a monotone increasing function of $(n \pi / \gamma)$, so the inequality (eq 15) is true.

In the two-dimensional potential $(\nu=-1 / 2)$

$$
H_{-1 / 2}(t)=i H_{1 / 2}(t)=\left(\frac{2}{\pi t}\right)^{1 / 2} e^{i t}
$$

and

$$
\begin{gathered}
H_{-3 / 2}^{(2)}(t)=-i H_{1 / 2}^{(2)}(t)=\left(\frac{2}{\pi t}\right)^{1 / 2} e^{-i t}, \\
J_{-1 / 2}(t)=\left(\frac{2}{\pi t}\right)^{1 / 2} \cos t
\end{gathered}
$$

and

$$
J_{1 / 2}(t)=\left(\frac{2}{\pi t}\right)^{1 / 2} \sin t
$$

so the analogues of eq 19a and 19b are

$$
\begin{aligned}
\pi\left(\frac{i n \pi}{\gamma}\right) H_{-1 / 2}\left(\frac{i n \pi}{\gamma}\right) J_{-1 / 2}\left(\frac{i n \pi}{\gamma}\right) & =1+e^{\frac{-2 n \pi}{\gamma}} \\
\pi\left(\frac{n \pi}{\gamma}\right) H_{-1 / 2}\left(\frac{i n \pi}{\gamma}\right) J_{1 / 2}\left(\frac{i n \pi}{\gamma}\right) & =1-e^{\frac{-2 n \pi}{\gamma}},
\end{aligned}
$$


the latter being also a monotone increasing function of $n \pi / \gamma$ as in eq 15 with +1 as upper limit.

For small values of $\gamma$ we may place the second member of eq 19a equal to 1, (as is very accurately the second member of eq (20a), which gives by eq 6 for both values of $\nu$,

$$
\begin{aligned}
& \mathrm{X}_{s k}^{(1)}=\frac{2}{\pi^{3}} \sum_{n=1}^{\infty}\left[n^{2}+\left(\frac{\gamma \alpha_{s}}{\pi}\right)^{2}\right] \cdot\left[n^{2}+\left(\frac{\gamma \alpha_{k}}{\pi}\right)^{2}\right] \\
& =\frac{2}{\pi^{3}\left(\alpha_{k}^{2}-\alpha_{s}^{2}\right)} \sum_{n=1}^{\infty}\left\{\frac{\alpha_{k}^{2}}{n\left[n^{2}+\left(\frac{\gamma \alpha_{k}}{\pi}\right)^{2}\right]}-\frac{\alpha_{s}^{2}}{n\left[n^{2}+\left(\frac{\gamma \alpha_{s}}{\pi}\right)^{2}\right]}\right\} \\
& =\frac{2}{\pi^{3}\left(\alpha_{k}^{2}-\alpha_{s}^{2}\right)}\left[\alpha_{k}^{2} S\left(\frac{\gamma \alpha_{k}}{\pi}\right)-\alpha_{s}^{2} S\left(\frac{\gamma \alpha_{s}}{\pi}\right)\right] \text {. }
\end{aligned}
$$

For $k=s$ this is $0 / 0$ but is determinate,

$$
X_{s s}^{(1)}=\frac{2}{\pi^{3}}\left[S\left(\frac{\gamma \alpha_{s}}{\pi}\right)-\left(\frac{\gamma \alpha_{s}}{\pi}\right)^{2} T\left(\frac{\gamma \alpha_{s}}{\pi}\right)\right],
$$

where $S(u)$ and $T(u)$ are defined below.

The expressions (eq 21a and 21b) are, for all practical purposes, exact in the two-dimensional case $\nu=-1 / 2$, but as shown by eq 19 a they may be in error for the case $\nu=0$ by a part in 2,000 when $\gamma$ is as large as $1 / 5$. However this corresponds to an error of 2 in $10^{6}$ in capacitance so that eq 21a and $21 \mathrm{~b}$ are sufficient in either case. To the same approximation, eq 18 becomes

$$
\begin{gathered}
\gamma \sum_{k=1}^{\infty} X_{s k}^{(1)}=\frac{\operatorname{coth} \gamma \alpha_{s}-\frac{1}{\gamma \alpha_{s}}}{2 \gamma \alpha_{s}} \\
S(u) \equiv \sum_{n=1}^{\infty} \frac{1}{n\left(n^{2}+u^{2}\right)}=\frac{1}{u^{2}}[0.57722+R \psi(1+i u)] \\
T(u)=-D_{u^{2}} S(u)=\sum_{1}^{\infty} \frac{1}{n\left(n^{2}+u^{2}\right)^{2}}
\end{gathered}
$$

where $\psi(z)=\frac{d}{d z} \log \Gamma(z)$. From the asymptotic expansion of the psi-function it is found that when $u$ is large $S(u)$ vanishes with the asymptotic expansion,

$$
S(u) \sim \frac{1}{u^{2}}\left[\frac{1}{2} \log \left(1+u^{2}\right)+0.57722-\frac{1}{2\left(1+u^{2}\right)}\right] .
$$

For value of $u$ as small as 1 this formula is in error by less than one percent.

We have to compute $S(u)$ and $T(u)$ for values of $u$ from $u$ slightly greater than zero up to $u=1$ or 2. For this purpose we may get more rapidly converging series by use of the identity

$$
\frac{1}{n\left(n^{2}+u^{2}\right)}=\frac{1}{n^{3}}-\frac{u^{2}}{n^{5}}+\frac{u^{4}}{n^{7}}-\frac{u^{6}}{n^{9}}+\frac{u^{8}}{n^{11}}-\frac{u^{10}}{n^{11}\left(n^{2}+u^{2}\right)}
$$

Since $\sum_{n=1}^{\infty} \frac{1}{n^{2 k+1}}$ is accurately known, we find

$S(u)=1.20206-1.03693 u^{2}+1.00835 u^{4}-$

$1.00201 u^{6}+1.00049 u^{8}-u^{10} \sum_{n=1}^{\infty} \frac{1}{n^{11}\left(n^{2}+u^{2}\right)}$ and

$$
\begin{array}{r}
T(u)=1.03693-2.01670 u^{2}+3.00602 u^{4}- \\
4 u^{6} \sum_{n=1}^{\infty} \frac{1}{n^{7}\left(n^{2}+u^{2}\right)^{2}}-3 u^{8} \sum_{n=1}^{\infty} \frac{1}{n^{9}\left(n^{2}+u^{2}\right)^{2}} .
\end{array}
$$

\section{Application to Capacitance}

The surface density of charge on the bottom of the hole or slot is a function of $\xi(=\rho / a)$, given by

$$
2 \pi a \sigma=\frac{a}{2}\left(D_{x} V\right)_{x=b}=\sum_{s=1}^{\infty} \frac{x_{s} \xi^{-\nu} J_{\nu}\left(\alpha_{s} \xi\right)}{\sinh \beta \alpha_{s} J_{\nu+1}\left(\alpha_{s}\right)} .
$$

The charge on this circular face of radius $a$ is

$$
Q=2 \pi \int_{0}^{a} \rho \sigma d \rho=a \sum_{s=1}^{\infty} \frac{x_{s}}{\alpha_{s} \sinh \beta \alpha_{s}} .
$$

The charge on the bottom of the slot, (whose width is $2 a$ ) and per cm length of slot, is

$$
Q^{\prime}=2 \int_{0}^{a} \sigma d \rho=\frac{1}{\pi} \sum_{s=1}^{\infty} \frac{x_{s}}{\alpha_{s} \sinh \beta \alpha_{s}} .
$$

The coefficient of capacitance, $C$, between the plane $x=0$ and the bottom of the hole is given (in $\mathrm{cm}$ ) by

$$
\frac{C}{a}=\sum_{s=1}^{\infty} \frac{x_{s}}{\alpha_{s} \sinh \beta \alpha_{s}} \text { where } J_{\rho}\left(\alpha_{s}\right)=0 .
$$


In the two-dimensional case, the coefficient $C^{\prime}$ per unit length of slot is given by

$$
\pi C^{\prime}=\sum_{s=1}^{\infty} \frac{x_{s}^{\prime}}{\alpha_{s}^{\prime} \sinh \beta \alpha_{s}^{\prime}} \text { where } \cos \alpha_{s}^{\prime}=0 .
$$

$\alpha_{s}^{\prime}=\left(s-\frac{1}{2}\right) \pi$

The coefficients, $x_{s}$, are solutions of eq 9 given by eq 17 and $x_{s}^{\prime}$ are similar functions of $\alpha_{s}^{\prime}$.

The two infinite, parallel planes with separation $c$ (fig. 1), bring about a uniform electric field at points between them, which are far from the edge $A_{2}$ of the hole. To approximate this with small planes, they must be close together. Hence one restriction which, in the nature of the case, must always be made is that $\gamma,(=c / a)$ is small. A second restriction that may be made, and which further simplifies the computations, is that the hole is not relatively shallow. There would be no hardship in practice if these restrictions are, roughly

$$
\begin{gathered}
0 \leqslant \gamma \equiv c / a \leqslant 1 / 5 \\
1 \leqslant \beta \equiv(b-c) / a<\infty .
\end{gathered}
$$

By reason of the first, the constants $x_{1}, x_{2}, x_{3}$ are of the order of magnitude of unity, but they are by no means of equal importance in the formula for capacitance. By reason of the second restriction, the denominators in eq 25 and $25 \mathrm{a}$ are such powerful convergence factors that the second term of of the series is less than 2 percent of the first, and any term is less than 4 percent of the term that precedes it. Only two or three terms of these series are required, and the accuracy depends mainly upon the precision with which the first constant $x_{1}$ is evaluated.

For this reason we retain in eq 17 only the first approximation

$$
x_{s}:=\frac{A_{s}}{\gamma \alpha_{s}} \text { for } s>1 .
$$

For the important constant $x_{1}$ we retain also the first series in eq 17

$$
x_{1}=\frac{A_{1}}{\gamma \alpha_{1}}\left(1+\epsilon_{1}\right),
$$

where

$$
\epsilon_{1}=\left(\gamma \alpha_{1}\right)^{2} \sum_{k=1}^{\infty} \frac{A_{k} X_{1 k}^{(1)}}{\alpha_{k}}
$$

$$
=\left(\gamma \alpha_{1}\right)^{2} \sum_{k=1}^{\infty} \frac{X_{1 k}^{(1)}}{\alpha_{k}\left(\operatorname{coth} \gamma \alpha_{k}+\operatorname{coth} \beta \alpha_{k}\right)}
$$

or by eq 21

where

$$
\epsilon_{1}=\frac{2}{\pi}\left(\frac{\gamma \alpha_{1}}{\pi}\right)^{2}\left[\sum_{k=1}^{n} P_{k}+R_{n+1}\right]
$$

$$
\begin{aligned}
& R_{n+1}=\sum_{k=n+1}^{\infty} P_{k} \\
& P_{1}=\frac{S\left(\frac{\gamma \alpha_{1}}{\pi}\right)-\left(\frac{\gamma \alpha_{1}}{\pi}\right)^{2} T\left(\frac{\gamma \alpha_{1}}{\pi}\right)}{\alpha_{1}\left(\operatorname{coth} \gamma \alpha_{1}+\operatorname{coth} \beta \alpha_{1}\right)}
\end{aligned}
$$

For $k>1$

$$
\begin{aligned}
& P_{k}=\frac{1}{\alpha_{k}\left(\operatorname{coth} \gamma \alpha_{k}+\operatorname{coth} \beta \alpha_{k}\right)} \cdot \\
& \left\{S\left(\frac{\gamma \alpha_{k}}{\pi}\right)-\frac{\alpha_{1}^{2}\left[S\left(\frac{\gamma \alpha_{1}}{\pi}\right)-S\left(\frac{\gamma \alpha_{k}}{\pi}\right)\right]}{\alpha_{k}^{2}-\alpha_{1}^{2}}\right\}
\end{aligned}
$$

When $\beta \geqslant 1$ we may place $\operatorname{coth} \beta \alpha_{k}=1$ for $k>$ 1 since coth $\alpha_{2}=1.000$. If we take $n$ just large enough to make $\gamma \alpha_{n} / \pi$ as large as $1 / 2$, then $\gamma \alpha_{n+1}$ is of the order of $3 / 2$, so that in the series defining $R_{n+1}$ we may neglect variations of $\operatorname{coth} \gamma \alpha_{k}$. Also the term in eq 30 with factor $\alpha_{1}^{2} /\left(\alpha_{k}^{2}-\alpha_{1}^{2}\right)$ will then be negligible compared with the first terms so that

$$
R_{n+1}=\frac{\gamma}{\pi\left(1+\operatorname{coth} \gamma \alpha_{n+1}\right)} \sum_{k=n+1}^{\infty} \frac{S\left(\gamma \alpha_{k} / \pi\right)}{\left(\gamma \alpha_{k} / \pi\right)} .
$$

We may estimate $\mathrm{R}_{n+1}$ by using eq 23 so

$$
\begin{aligned}
& 2 \pi\left(1+\operatorname{coth} \gamma \alpha_{n+1}\right) R_{n+1}=\gamma \sum_{k=n+1}^{\infty} f\left(\frac{\gamma \alpha_{k}}{\pi}\right) \\
& f(x) \equiv \frac{1}{x^{3}}\left[\log \left(1+x^{2}\right)+1.1544-\frac{1}{1+x^{2}}\right],
\end{aligned}
$$

where $f(x)$, defined by this equation, is a positive, monotone function of $x$, which decreases slowly with increasing $x$.

At this point it becomes necessary to distinguish between the two cases.

In the three-dimensional case $(\nu=0)$

$$
\alpha_{k} / \pi \sim k-\frac{1}{4}
$$

very approximately, so

$$
2 \pi\left(1+\operatorname{coth} \gamma \alpha_{n+1}\right) R_{n+1}=\gamma \sum_{k=n+1}^{\infty} f\left(\gamma\left[k=\frac{1}{4}\right]\right),
$$


in the case $\nu=0$.

Also $\alpha_{k} / \pi=k-\frac{1}{2}$ exactly, in the case $\nu=-\frac{1}{2}$, so that

$$
2 \pi\left(1+\operatorname{coth} \gamma \alpha_{n+1}\right) R_{n+1}=\gamma \sum_{k=n+1}^{\infty} f\left(\gamma\left[k-\frac{1}{2}\right]\right)
$$

in the case $\nu=-\frac{1}{2}$.

The numerical value of the sum of a convergent series of positive terms which continually decrease, say

$$
A=\sum_{k=n+1}^{\infty} F(k),
$$

represents the sum of the areas of the rectangles each of unit width and height $F(k)$.

The positive, monotone function of $x$,

$$
y=F(x-1 / 2),
$$

represents a smooth curve that passes through the mid-point of the top of each rectangle.

When, as in the present case, this function decreases slowly with increasing $x$, the area under this curve from $x=n+1$ to $x=\infty$ is a good approximation to the value $A$ of the series, so that

$$
\begin{aligned}
A & =\sum_{k=n+1}^{\infty} F(k) \\
& =\int_{x=n+1}^{\infty} F\left(x-\frac{1}{2}\right) d x=\int_{x=n+\frac{1}{2}}^{\infty} F(x) d x .
\end{aligned}
$$

Taking $F(k)=\gamma f\left(\frac{\gamma \alpha}{\pi} k\right)$ gives

where

$$
\left.\begin{array}{c}
\gamma \sum_{k=n+1}^{\infty} f\left(\frac{\gamma \alpha}{\pi} k\right)=\int_{\gamma\left(\frac{\alpha_{n}}{\pi}+\frac{1}{2}\right)}^{\infty} f(x) d x \\
\frac{\alpha_{n}}{\pi}+\frac{1}{2} \sim n+\frac{1}{4} \text { if } J_{0}\left(\alpha_{k}\right)=0 \\
=n \text { if } \cos \alpha_{k}=0,
\end{array}\right\}
$$

provided $f(x)$ is a positive, monotone decreasing function of $x$ for

$$
x>\gamma\left(\frac{\alpha_{n}}{\pi}+\frac{1}{2}\right) .
$$

Hence from the function $f(x)$ in eq 31 we obtain

$$
R_{n+1} \sim \frac{1}{4 \pi\left(1+\operatorname{coth} \gamma \alpha_{n+1}\right)}\left\{\frac{0.1544+\mathrm{l}\left(\log +x_{n}^{2}\right)}{x_{n}^{2}}+\right.
$$

$\left.2 \log \left(\frac{1+x_{n}^{2}}{x_{n}^{2}}\right)\right\}$,

where

$$
x_{n}=\gamma\left(\frac{\alpha_{n}}{\pi}+\frac{1}{2}\right) \text {. }
$$

Equations 25 and 25a for capacitance become

$$
\frac{C}{a}=\frac{1}{\gamma} \sum_{s=1}^{\infty} \frac{\left(1+\epsilon_{s}\right) \sinh \gamma \alpha_{s}}{\alpha_{s}^{2} \sinh (\beta+\gamma) \alpha_{s}} \text { where } J_{\sigma}\left(\alpha_{s}\right)=0
$$

$\pi C^{\prime}=\frac{1}{\gamma} \sum_{s=1}^{\infty} \frac{\left(1+\epsilon_{s}^{\prime}\right) \sinh \gamma \alpha_{s}^{\prime}}{\left(\alpha_{s}^{1}\right)^{2} \sinh (\beta+\gamma) \alpha_{s}^{\prime}}$ where $\cos \alpha_{s}^{\prime}=0$

$\boldsymbol{\epsilon}_{s}, \boldsymbol{\epsilon}_{s}^{\prime}=0$ if $s>1, \gamma \equiv c / a$ and $\beta \equiv(b-c) / a$

$\epsilon_{1}, \epsilon_{1}^{\prime}$ computed by eq 24, 28, 29, 30, and 33 .

From an examination of the effects of retaining all terms in the second members of eq 19a and $19 \mathrm{~b}$ it is easy to ascertain that the error in eq 35 , whatever it is, must be of the same order of magnitude as that in eq $35^{\prime}$. The terms of eq 19a and $19 \mathrm{~b}$, which are neglected, are estimated to produce an alteration less than 2 in $10^{5}$ in capacitance. The error in eq 35 and 35 a increases with $\gamma$, vanishing with it, but is practically independent of $\beta$, for $1 \leqslant \beta<\infty$. The computed capacitance will be lower than the correct value.

To estimate the error we apply eq $35 \mathrm{a}$ to compute $\pi \mathrm{C}^{\prime}$ for the case of a standard capacitor constructed here, which approximates to the two-dimensional type. Its dimensions are

$$
\left.\begin{array}{rl}
a & =0.8540 \mathrm{~cm} \\
c & =0.2000 \mathrm{~cm} \\
b-c & =1.8940 \mathrm{~cm}
\end{array}\right\} \text { so }\left\{\begin{array}{l}
\gamma=\frac{c}{a}=0.23419 \\
\beta=(b-c) / a=2.2178
\end{array}\right.
$$

By eq 28, taking $n=4$,

$$
\begin{gathered}
\boldsymbol{\epsilon}_{1}=0.00873\left[\sum_{k=1}^{4} P_{k}+R_{5}\right] \\
P_{1}=0.194_{5} \\
P_{2}=0.101_{5} \\
P_{3}=0.057_{2} \\
P_{4}=0.034_{8} \\
R_{5}=0.096_{1}
\end{gathered}
$$

$$
0.484=\sum_{k=1}^{4} P_{k}+R_{\overline{5}}
$$


This gives $\epsilon_{1}=0.00423$.

It was found that with $n=10$, the sum $\left[\sum_{k=1}^{10} P_{k}+\right.$ $\left.R_{11}\right]$ gave a value of $\epsilon_{1}$ so near this as to affect the capacitance by less than two parts in a millon.

The result of computing $\pi C^{\prime}$ by eq $35 \mathrm{a}$ is to be compared with that given by the exact equation (eq 48) to be derived below. The constant $\xi_{0}$ appearing in eq 48 is given by eq $43 \mathrm{a}$, which gives $\xi_{0}=\frac{2}{\pi} \tan \gamma=0.146454_{4}$.

\begin{tabular}{|c|c|c|}
\hline & \multicolumn{2}{|c|}{$\pi C^{\prime}$} \\
\hline . & by eq $35 a$ & by eq 48 \\
\hline $\begin{array}{l}\text { 1st term } \\
2 \mathrm{~d} \text { term } \\
3 \mathrm{~d} \text { term } \\
\pi C^{\prime}\end{array}$ & $\begin{array}{r}0.027795 \\
.000005 \\
.000000 \\
.027800\end{array}$ & $\begin{aligned} 0.0278108 \\
.0000037 \\
.00000000 \\
.0278145\end{aligned}$ \\
\hline
\end{tabular}

Equation 35a here gives $C^{\prime}$ too low by one in 2,000 .

Another computation for a shallower slot, $\beta=1$, with the same $\gamma$ as above gives a coefficient of capacitance $C^{\prime}$ about seven times as large.

\begin{tabular}{|c|c|c|}
\hline & \multicolumn{2}{|c|}{$\pi C^{\prime}$} \\
\hline & by eq $35 a$ & by eq 48 \\
\hline $\begin{array}{l}1 \text { st term } \\
2 \mathrm{~d} \text { term } \\
\text { 3d term } \\
\pi C^{\prime}\end{array}$ & $\begin{array}{r}0.192142 \\
.001537 \\
.000026 \\
.193707\end{array}$ & $\begin{array}{r}0.192688 \\
.001146 \\
.000000 \\
.193834\end{array}$ \\
\hline
\end{tabular}

Hence we expect the pacitance in case of the cylindrical hole computed by eq 35 will also be lower than the correct value by about one in 2,000 (if $\gamma=0.2$ ). This is probably less than the experimental error of the comparison of such very small capacitors.

If a precision greater than one in 2,000 were required, this might be found by retaining one more series in eq 17 for $x_{1}$, that is,

$$
\begin{aligned}
x_{1}= & \frac{A_{1}}{\gamma \alpha_{1}}\left[1+\left(\gamma \alpha_{1}\right)^{2} \sum_{k=1}^{\infty} \frac{A_{k} X_{1 k}^{(1)}}{\alpha_{k}}+\gamma^{4} \alpha_{1}^{2} \sum_{k=1}^{\infty} \frac{A_{k} X_{1 k}^{(2)}}{\alpha_{k}}\right]= \\
& \frac{A_{1}}{\gamma \alpha_{1}}\left[1+\epsilon_{1}+\delta_{1}\right]
\end{aligned}
$$

where

$$
\delta=\gamma^{4} \alpha_{1}^{2} \sum_{k=1}^{\infty} \frac{A_{k} X_{1 k}^{(2)}}{\alpha_{k}}=\gamma^{4} \alpha_{1}^{2} \sum_{k=1}^{\infty} \frac{A_{k}}{\alpha_{k}} \sum_{s=1}^{\infty} \alpha_{s} A_{s} X_{1 s}^{(1)} X_{s k}^{(1)},
$$

where $X_{s k}^{(1)}$ is given by eq $21 \mathrm{a}, A_{k}$ by eq 8 .

For $\gamma=1 / 5$ eq 35 gives values of $C$ that are too small by one in 500 neglecting both $\epsilon_{1}$ and $\delta_{1}$; and too small by one in 2,000 neglecting $\delta_{1}$ only. From the computations given it appears that $\delta_{1}$ is about $1 / 8$ of $\epsilon_{1}$. The approximate evaluation of $\delta_{1}$ by this double series would require an evaluation of each remainder, as in the single series for $\epsilon_{1}$. It would require much labor for small gain.

An alternative estimate would be given by making a plot of the error in eq 35a as a function of $\gamma$ from $\gamma=0.1$ to 0.5 all with $\beta=1$. This could be taken as an estimate of the error in eq 35 .

\section{The Two-Dimensional Potential}

Let $\phi(x, \rho)$ be the two-dimensional potential that vanishes on the plane $x=0$ and has the constant value $\pi$ on the guard-conductor and on the base of the slot. Its conjugate function $\psi(x, \rho)$ is zero on the $x$-axis. Let $\psi_{1}$ and $\psi_{2}$ denote the value of $\psi_{1}$ at the points $A_{1}$ and $A_{2}$ respectively. The capacitance $C^{\prime}$ per unit length of slot is proportional to $\psi$, for the surface density $\sigma$ of charge on the base of the slot is given by

$$
4 \pi \sigma=\left[\frac{\partial \phi(x, \rho)}{\partial x}\right]_{x=b}=\frac{\partial}{\partial \rho} \psi(b, \rho),
$$

so the total charge $Q^{\prime}$ upon the base of the slot, (of width $2 a$ ), per cm length of slot is

$$
Q^{\prime}=\int_{-a}^{+a} \sigma(\rho) d \rho=\frac{1}{2 \pi} \int_{0}^{a} \frac{\partial \psi(b, \rho)}{\partial \rho} d \rho=\frac{\psi}{2 \pi} .
$$

The coefficient of capacitance $C^{\prime}$ per cm length of slot between the plane $x=0$ and the bottom of the slot is $C^{\prime}=\frac{Q^{\prime}}{\pi}(\mathrm{cm})$ or $\pi C^{\prime}=\frac{\psi_{1}}{2 \pi}$.

To find $\psi_{1}$ we first obtain the complex potential

$$
\omega=\phi+i \psi,
$$

which may be considered a function $\omega(z)$ of the complex variable $z=x+i \rho$.

To do this, the dielectric region $(D)$ of figure $2 \mathrm{a}$ is first represented conformally upon the 


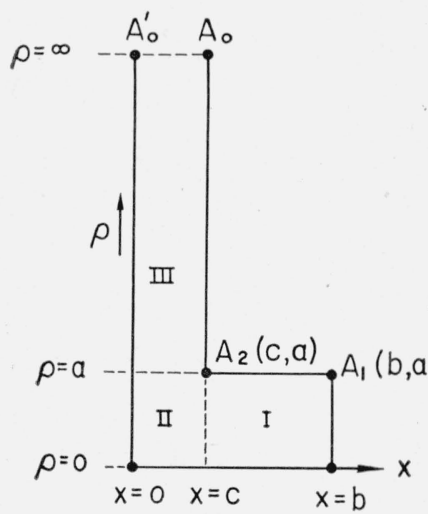

A

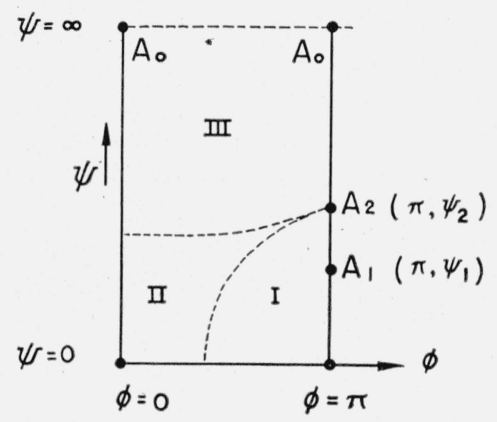

C

Figure 2. Dielectric volume.

A, Plane of $Z=X+i \rho ; B$, plane of $t=r e^{i \theta}$, eq $38 ; C$, potential strip $\omega=\phi+i \psi($ eq 47$)$.

quarter-annulus of figure $2 \mathrm{~b}$ defined by the plane polar coordinates

$$
1<r<\frac{1}{q} \text { and } 0<\theta<\frac{\pi}{2} .
$$

The conformance is indicated by similar lettering in figures 2 , a and $2, \mathrm{~b}$. This region $(D)$ may also be represented on the semi-infinite, potentialstrip as shown in figure 2, c where

$$
0<\phi<\pi \text { and } 0<\psi<\infty \text {. }
$$

The mapping equation we take is equivalent to

$$
\frac{\pi z}{c}=\frac{4 a i \zeta}{c}+i \log \frac{\vartheta_{1}\left(\zeta_{0}+\zeta\right) \vartheta_{2}\left(\zeta_{0}+\zeta\right)}{\vartheta_{1}\left(\zeta_{0}-\zeta\right) \vartheta_{2}\left(\zeta_{0}-\zeta\right)},
$$

and the potential is equivalent to

$$
\begin{aligned}
\omega & =i \log \frac{\vartheta_{1}\left(\zeta_{0}+\zeta\right) \vartheta_{2}\left(\zeta_{0}-\zeta\right)}{\vartheta_{1}\left(\zeta_{0}-\zeta\right) \vartheta_{2}\left(\zeta_{0}+\zeta\right)} \\
& =i \log \frac{\operatorname{sn}\left(u_{0}+u\right) c n\left(u_{0}-u\right)}{c n\left(u_{0}+u\right) \operatorname{sn}\left(u_{0}-u\right)}\left(\text { where } u=\frac{2 K}{\pi} \zeta\right)
\end{aligned}
$$

One is led to the equation of transformation by well-known methods, but for convenience of numerical computation, it is better to use the series that are equivalent to eq 37 and $37 \mathrm{a}$. By this method the discussion of the equation of transformation and of the potential may be made without any reference to, or knowledge of, thetafunctions or elliptic functions.

Accordingly we take a complex variable,

$$
t=r e^{i \theta}=e^{2 i \zeta}=e,{ }^{i \pi u / K}
$$

and start with the equation of transformation,

$$
\begin{aligned}
\frac{\pi}{a}(z-b)= & 2 \log (q t)-\gamma\left\{i \log \frac{\left(t-e^{i \theta_{0}}\right)\left(t+e^{i \theta_{0}}\right)}{\left(t-e^{-i \theta_{0}}\right)\left(t+e^{-i \theta_{0}}\right)}+\right. \\
& \left.4 \sum_{n=1}^{\infty} \frac{q^{4 n}\left(t^{-2 n}-t^{2 n}\right)}{2 n\left(1-q^{4 n}\right)} \sin 2 n \theta_{0}\right\}, \quad \text { (38a) }
\end{aligned}
$$

where the positive constants, $0<q<1$, and the angle $\theta_{0},\left(0<\theta_{0}<\frac{\pi}{2}\right)$, are to be found in terms of the given constants

$$
\gamma \equiv \frac{c}{a} \text { and } \beta \equiv \frac{b-c}{a} .
$$

Since $1<|t|=r<\frac{1}{q}$ in figure $2 \mathrm{~b}$ the logarithm in eq 38 a may be expanded in a series so that

$$
\begin{aligned}
\frac{\pi}{a}(z-b)= & 2 \log (q t)- \\
& 4 \gamma \sum_{n=1}^{\infty} \frac{q^{2 n}\left[(q t)^{-2 n}-(q t)^{2 n}\right]}{2 n\left(1-q^{4 n}\right)} \sin 2 n \theta_{0},
\end{aligned}
$$

which converges within and on the boundaries of the region $(D)$ of figure $2 \mathrm{~b}$. Its term-by-term derivative converges within, but not on the are $r=1$. To obtain a series for $\frac{d z}{d t}$ convergent on the boundaries, eq 38a may be differentiated, giving

$$
\begin{aligned}
\left(\frac{\pi t}{2 a}\right) \frac{d z}{d t}= & 1+\gamma\left[\frac{4 t^{2} \sin \theta_{0} \cos \theta_{0}}{\left(t^{2}+1\right)^{2}-4 t^{2} \cos ^{2} \theta_{0}}+\right. \\
& \left.2 \sum_{n=1}^{\infty} \frac{q^{4 n}\left(t^{-2 n}+t^{2 n}\right)}{1-q^{4 n}} \sin 2 n \theta_{0}\right] .
\end{aligned}
$$

To establish the correspondence for the point $A_{2}$ of figure $2 \mathrm{a}$ and figure $2 \mathrm{~b}$, we place $z=c+i a$ and and $t=e^{\frac{\pi}{2}}$ in eq 38a. This gives

$$
q=e^{-\frac{\pi}{2}\left\lfloor\beta+\frac{2 \gamma}{\pi} \theta_{0}\right]} .
$$


Also, since the point $A_{2}$ is the only point where angles are not preserved by this transformation, it is necessary that $d z / d t$ be either zero or infinite there. Since $z$ is continuous there, $d z / d t$ must vanish. Hence the second member of eq 39 must vanish when $t=e^{i \pi / 2}$. That is

$$
\tan \theta_{0}+4 \sum_{n-1}^{\infty} \frac{(-1)^{n+1} q^{4 n} \sin 2 n \theta_{0}}{1-q^{4 n}}=\frac{1}{\gamma} .
$$

These two equations determine $q$ and $\theta_{0}$ as functions of $\beta$ and $\gamma$.

If we write

and

$$
\left.\begin{array}{c}
\frac{\pi}{2} \xi_{0}=\frac{\pi}{2}-\theta_{0} \text { so } 0<\xi_{0}<1 \\
\gamma=\tan \delta
\end{array}\right\}
$$

then, eliminating $q$ between eq $40 \mathrm{a}$ and $40 \mathrm{~b}$ gives the following equation to determine $\xi_{0}$

$$
\cot \frac{\pi \xi_{0}}{2}+4 \sum_{n=1}^{\infty} \frac{e^{-2 n \pi\left(\beta+\gamma-\gamma \xi_{0}\right)} \sin n \pi \xi_{0}}{1-e^{-2 n \pi\left(\beta+\gamma-\gamma \xi_{0}\right)}}=\cot \delta .
$$

A very accurate solution of this for the case in which the slot is not relatively shallow ( $\beta$ not small) may be obtained by using a few terms of this series.

Thus it is found that

$$
\begin{aligned}
& \frac{\pi}{2}-\theta_{0}=\frac{\pi \xi_{0}}{2}=\delta+8 \sin ^{3} \delta \cos \delta e^{-2 \pi\left[\beta+\left(1-\frac{2 \delta}{\pi}\right) \tan \delta\right]} . \\
& \left\{1+3 C_{1} e^{-2 \pi\left[\beta+\left(1-\frac{2 \delta}{\pi}\right) \tan \delta\right]}+C_{2} e^{-4 \pi\left[\beta+\left(1-\frac{2 \delta}{\pi}\right) \tan \delta\right]}\right\}
\end{aligned}
$$

where $C_{1}=1+4 \sin ^{2} \delta-\frac{8}{3} \sin ^{3} \delta \cos \delta+\frac{16}{3} \sin ^{4} \delta$.

Also $\left|C_{2}\right|<32$, so the term in $C_{2}$ is negligible when $\beta \geqslant 1$ and $\delta$ is small.

When $\theta_{0}$ or $\xi_{0}$ is computed by this formula, the parameter $q$ is then computed by

$$
q=e^{-\pi\left(\beta+\gamma-\gamma \xi_{0}\right) / 2} .
$$

Since $t=r e^{i \theta}$, eq $38_{b}$ gives $x$ and $\rho$ as functions of $r$ and $\theta$ by series that converge in $(D)$, and on its boundaries

$$
\begin{aligned}
& \frac{x}{a}=\frac{b}{a}+\frac{2}{\pi} \log (q r)- \\
& \quad \frac{2 \gamma}{\pi} \sum_{n=1}^{\infty} \frac{q^{2 n}\left[(q r)^{-2 n}-(q r)^{2 n}\right]}{n\left(1-q^{4 n}\right)} \sin 2 n \theta_{0} \cos 2 n \theta
\end{aligned}
$$

$\frac{\rho}{a}=\frac{2 \theta}{\pi}+\frac{2 \gamma}{\pi} \sum_{n=1}^{\infty} \frac{q^{2 n}\left[(q r)^{-2 n}+(q r)^{2 n}\right]}{n\left(1-q^{4 n}\right)} \sin 2 n \theta_{0} \sin 2 n \theta$

$$
\text { for } 1 \leqslant r \leqslant \frac{1}{q} \text { and } 0 \leqslant \theta \leqslant \pi / 2 \text {. }
$$

The complex potential is

$$
\omega=\pi-4 \sum_{n=1}^{\infty} \frac{q^{2 n-1}\left[(q t)^{-(2 n-1)}-(q t)^{2 n-1}\right]}{(2 n-1)\left(1-q^{4 n-2}\right)} \sin (2 n-1) \theta_{0},
$$

which resolves into the two real equations

$$
\begin{aligned}
\phi= & \pi-4 \sum_{n=1}^{\infty} \frac{q^{2 n-1}\left[(q r)^{-(2 n-1)}-(q r)^{2 n-1}\right]}{(2 n-1)\left(1-q^{4 n-2}\right)} \\
& \sin (2 n-1) \theta_{0} \cos (2 n-1) \theta \\
\psi= & 4 \sum_{n=1}^{\infty} \frac{q^{2 n-1}\left[(q r)^{-(2 n-1)}+(q r)^{2 n-1}\right]}{(2 n-1)\left(1-q^{4 n-2}\right)} \\
& \sin (2 n-1) \theta_{0} \sin (2 n-1) \theta .
\end{aligned}
$$

The constant $\psi_{1}$ (at $A_{1}$ ) corresponds to $r=$ $\frac{1}{q}, \theta=\frac{\pi}{2}$.

The constant $\psi_{2}$ (at $A_{2}$ ) corresponds to $r=$ $1, \theta=\frac{\pi}{2}$.

If these two positive constants are determined thus, the equation that maps the region $(D)$ of the z-plane conformally upon the semi-infinite strip of figure $2 \mathrm{c}$ is

$$
z=\frac{c}{\pi} \int_{0}^{\omega} \sqrt{\frac{\cos \omega^{\prime}+\cosh \psi_{2}}{\cos \omega^{\prime}+\cosh \psi_{1}}} d \omega^{\prime} .
$$

To prove this we take the complementary modulus of the elliptic function of $u$ in eq 37 as

$$
k^{\prime}=\frac{\tanh \frac{\psi_{2}}{2}-\tanh \frac{\psi_{1}}{2}}{\tanh \frac{\psi_{2}}{2}+\tanh \frac{\psi_{1}}{2}}
$$

and determine the constant $u_{0}$ by

$\frac{s n u_{0} c n u_{0}}{d n u_{0}}=\frac{1}{2}\left(\tanh \frac{\psi_{2}}{2}+\tanh \frac{\psi_{1}}{2}\right)$ where $0<u_{0}<\frac{K}{2}$.

Details are omitted as we do not make use of the relation (eq 47).

From eq $46 \mathrm{~b}$ we obtain (placing $r=\frac{1}{q}$ and $\theta=\frac{\pi}{2}$ ) 


$$
\frac{\psi_{1}}{2 \pi}=\frac{4}{\pi} \sum_{n=1}^{\infty} \frac{(-1)^{n+1} q^{2 n-1} \sin (2 n-1) \theta_{0}}{(2 n-1)\left(1-q^{4 n-2}\right)} .
$$

Hence the coefficient $C^{\prime}$ of capacitance per unit sngth of slot is given by

$$
\pi C^{\prime}=\frac{4}{\pi} \sum_{n=1}^{\infty} \frac{(-1)^{n+1} q^{2 n-1} \sin (2 n-1) \theta_{0}}{(2 n-1)\left(1-q^{4 n-2}\right)}
$$

Or letting $\alpha_{s}^{\prime}=(2 s-1) \frac{\pi}{2}$ this may be written

$$
\pi C^{\prime}=\sum_{s=1}^{\infty} \frac{\cos \alpha_{s}^{\prime} \xi_{0}}{\alpha_{s}^{\prime} \sinh \alpha_{s}^{\prime}\left(\beta+\gamma-\gamma \xi_{0}\right)},
$$

where $\xi_{0}$ is the root of eq 42 , which may be computed by eq 43a. This equation gives $C^{\prime}$ with all the precision that may be desired. This is the equation which may be used to find the error in eq 35a which would be of the same order as that in the three-dimensional formula (eq 35).

The deeper the slot, the more rapid is the convergence of the series (eq 48). Although this series converges for any slot however shallow, it becomes practically useless for computation when $\beta$ is small.

To obtain a formula of complementary nature to eq 48, whose rate of convergence gets more rapid as the slot gets more shallow we may make use of a transformation, which introduces a parameter $q_{1}$, which is found to be equal to $\sqrt{q^{\prime}}$.

In this case we transform the dielectric region of figure 2, a, upon the semicircular annulus of a $t$ plane, as shown in figure 3.

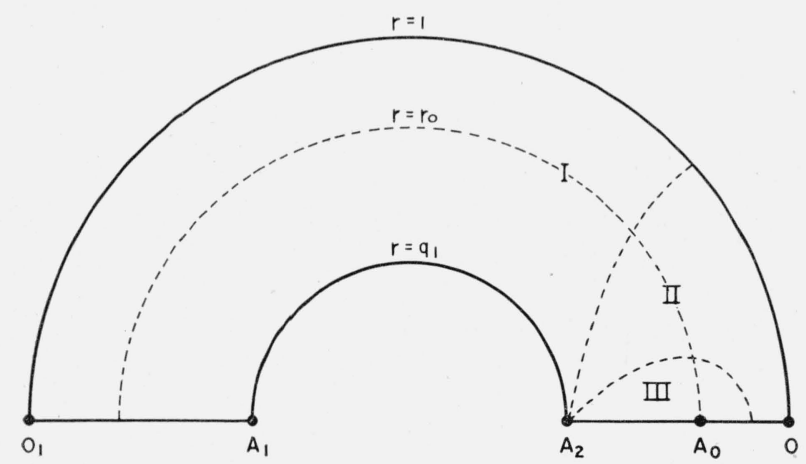

Figure 3. Semicircular annulus for eq 49.

As in the preceding case $r$ and $\theta$ are polar coordinates and

$$
t=r e^{i \theta},
$$

but in figure 3 the range of $r$ and $\theta$ is

$$
q_{1}<r<1 \text { and } 0<\theta<\pi .
$$

The equation of transformation is now

$$
\begin{aligned}
& \frac{i \pi z}{c}=\left(\frac{b-c}{c}\right) \log t+\log \left(\frac{t-r_{0}}{1-r_{0} t}\right)+ \\
& \sum_{n=1}^{\infty} \frac{q_{1}^{2 n}\left(r_{0}^{-n}-r_{0}^{n}\right)\left(t^{-n}-t^{n}\right) .}{n\left(1-q_{1}^{2 n}\right)} .
\end{aligned}
$$

The positive constants $q_{1}$ and $r_{0}$ where $0<q_{1}<$ $r_{0}<1$ are determined by securing the correspondence at $A_{2}$ and the vanishing of $d Z / d t$ at $A_{2}$ where angles are not preserved. These two equations are

$$
2 \sum_{n=1}^{\infty}\left(\frac{q_{1}}{r_{0}}\right)^{n} \cdot\left(\frac{1-r_{0}^{2 n}}{1-q_{1}^{2 n}}\right)=\frac{b-c}{c}=\frac{\beta}{\gamma}
$$

and

$$
q_{1}^{\beta}=e^{-\pi} r_{0}^{-\gamma}
$$

$$
\text { Let } \left.\begin{array}{rl}
u_{1} & =\log \frac{1}{q_{1}} \text { or } q_{1}=e^{-u_{1}} \\
u_{0} & =\log \frac{1}{r_{0}} \text { or } r_{0}=e^{-u_{0}} \cdot
\end{array}\right\}
$$

Then the two equations (eq 50a and 50b) may be written

$$
2 \sum_{n=1}^{\infty} \frac{\sinh n u_{0}}{\sinh n u_{1}}=\beta / \gamma
$$

and

$$
u_{0}=\frac{\pi-\beta u_{1}}{\gamma}
$$

Hence, eliminating $u_{0}$, the equation that determines $u_{1}$ is

$$
2 \sum_{n=1}^{\infty} \frac{\sinh n\left(\pi-\beta u_{1}\right) / \gamma}{\sinh n u_{1}}=\frac{\beta}{\gamma} .
$$

The complex potential is

$$
\begin{aligned}
\omega & =\phi+i \psi \\
& =-i \log \left(\frac{t-r_{0}}{1-r_{0} t}\right)+i \sum_{n=1}^{\infty} \frac{q_{1}^{2 n}\left(r_{0}^{-n}-r_{0}^{n}\right)\left(t^{-n}-t^{n}\right)}{n\left(1+q_{1}^{2 n}\right)} \\
& =\frac{\pi z}{c}+i\left(\frac{b-c}{c}\right) \log t+2 i \sum_{n=1}^{\infty} \frac{q_{1}^{2 n}\left(r_{0}^{-n}-r_{0}^{n}\right)\left(t^{-n}-t^{n}\right)}{n\left(1-q_{1}^{4 n}\right)} .
\end{aligned}
$$

At the point $\mathrm{A}_{1}, \frac{\pi z}{c}=\frac{\pi b}{c}+\frac{i \pi a}{c}, \phi=\pi, \psi=\psi_{1}, t=q_{1} e^{i \pi}$

$$
=-q_{1} \text {. }
$$

Equation 53 gives 


$$
\begin{aligned}
\psi_{1} & =\frac{\pi a}{c}+\left(\frac{b-c}{c}\right) \log q_{1}-2 \sum_{n=1}^{\infty} \frac{(-1)^{n+1} q_{1}^{n}\left(r_{0}^{-n}-r_{0}^{n}\right)}{n\left(1+q_{1}^{2 n}\right)} \\
& =\frac{\pi a}{c}-\frac{\beta}{\gamma} u_{1}-2 \sum_{n=1}^{\infty} \frac{(-1)^{n+1} \sinh n u_{0}}{n \cosh n u_{1}}
\end{aligned}
$$

From this we obtain the following equivalent of eq 48:

$\pi C^{\prime}=\frac{a}{2 c}-\frac{1}{2 \pi}\left[\frac{\beta u_{1}}{\gamma}+2 \sum_{n=1}^{\infty} \frac{(-1)^{n+1} \sinh n\left(\pi-\beta u_{1}\right) / \gamma}{n \cosh n u_{1}}\right]$,

where $u_{1}$ is a function of $\beta$ and $\gamma$ determined by eq 52c. This may also be written

$\pi C^{\prime}=\frac{a}{2 c}-\frac{1}{2 \pi}\left[\frac{\beta}{\gamma} \log \frac{1}{q_{1}}+2 \sum_{n=1}^{\infty} \frac{(-1)^{n+1} q_{1}^{n}\left(r_{0}^{-n}-r_{0}^{n}\right)}{n\left(1+q_{1}^{2 n}\right)}\right]$

where $r_{0}$ and $q_{1}$ are determined by eq 50a and 50b. For a relatively shallow slot in which $\beta / \gamma$ is small, while $\pi / \gamma$ is neither very small nor very large, we find to the second order inclusive in $\beta$ or $q_{1}$

$$
q_{1}=\frac{\beta}{2 \gamma}\left(1-\frac{\beta}{2 \gamma} \operatorname{coth} \frac{\pi}{\gamma}\right) \frac{r_{0}}{1-r_{0}^{2}},
$$

where

$\log r_{0}=-\left[\frac{\pi}{\gamma}+\left(\frac{\beta}{\gamma+\beta \operatorname{coth} \pi / \gamma}\right) \log \frac{\beta\left(1-\frac{\beta}{2 \gamma} \operatorname{coth} \frac{\pi}{\gamma}\right)}{4 \gamma \sinh \pi / \gamma}\right]$.

This applies for a slot so shallow that formula (eq 48) would be impracticable.

\section{Appendix}

Formulation of the potential as Contour Integrals.

The generalized potential equation (where $\nu=0$ or $\nu=-1 / 2)$,

$$
\left(D_{x}^{2}+D_{\rho}^{2}\right) V+\frac{1+2 v}{\rho} D_{\rho} V=0,
$$

has solutions of the form

$$
V=\left(\left(C_{1} \cosh \frac{t x}{a}+C_{2} \sinh \frac{t x}{a}\right) \xi^{-\nu} C_{\nu}(t \xi),\right.
$$

where $\xi=\rho / a$ and $C_{\nu}$ is any cylinder function of $t \xi$ with parameter $\nu$. The constant $t$ is arbitrary and may be complex. The two fundamental equa- tions, which are satisfied by all cylinder functions, will be needed for reference. They are

$$
\left.\begin{array}{l}
C_{\nu-1}(t \xi)-C_{\nu+1}(t \xi)=2 C_{v}^{\prime}(t \xi) \\
C_{\nu-1}(t \xi)+C_{\nu+1}(t \xi)=\frac{2 \nu}{t \xi} C_{\nu}(t \xi),
\end{array}\right\}
$$

which are equivalent to

$$
\left.\begin{array}{l}
D_{\xi}\left(\xi^{\nu} C_{\nu}(t \xi)\right)=t \xi^{\nu} C_{\nu-1}(t \xi) \\
D_{\xi}\left(\xi^{-\nu} C_{\nu}(t \xi)\right)=t \xi^{-\nu} C_{\nu+1}(t \xi) .
\end{array}\right\}
$$

The cylinder functions here used are Bessel's function $J_{\nu}(t)$ and the two Hankel's fanctions $H_{\nu}^{(1)}(t)$ and $H_{\nu}^{(2)}(t)$. However, the latter will only be used in passing, so that since $H_{\nu}^{(1)}$ occurs frequently it may be denoted by $H_{\nu}(t)$.

From the identity in $t$,

$$
H_{\nu}(t) J_{\nu}^{\prime}(t)-H_{\nu}^{\prime}(t) J_{\nu}(t)=\frac{2}{i \pi t},
$$

we obtain from the fundamental equations (eq 3) for the case $t=\alpha_{s}$, where $J_{\nu}\left(\alpha_{s}\right)=0$,

$$
\begin{aligned}
J_{\nu}^{\prime}\left(\alpha_{s}\right) & =-J_{\nu+1}\left(\alpha_{s}\right) \text { and } i \pi \alpha_{s} H_{\nu}\left(\alpha_{s}\right) \\
& =-2 / J_{\nu+1}\left(\alpha_{s}\right) \\
\frac{i \pi t H_{\nu}(t)}{2 J_{\nu}^{\prime}(t)} & =\frac{1}{J_{\nu}^{2}\left(\alpha_{s}\right)} \text { when } t=\alpha_{s} .
\end{aligned}
$$

In all that follows it is understood that the $t$-plane is cut along the negative real axis so that $-\pi<\arg t<\pi$.

There is the identical relation

$$
2 J_{\nu}(t)=H_{\nu}(t)+H_{\nu}^{(2)}(t) .
$$

The circuital relations around the branch-point $t=0$ are

$H_{\nu}^{(2)}\left(t e^{-i \pi}\right)=-e^{i_{\nu} \pi} H_{\nu}(t)$ and $J_{\nu}\left(t e^{ \pm i \pi}\right)=e^{ \pm i_{\nu \pi}} J_{\nu}(t)$.

When $|t|$ is large

and

$$
\left.\begin{array}{c}
H_{\nu}(t) \sim \sqrt{\frac{2}{\pi t}} e^{i\left(t-\left[\nu+\frac{1}{2}\right] \frac{\pi}{2}\right)} \\
H_{\nu}^{(2)}(t) \sim \sqrt{\frac{2}{\pi t}} e^{-i\left(t-\left[\nu+\frac{1}{2}\right] \frac{\pi}{2}\right)} \cdot
\end{array}\right\}
$$


In certain cases the cut in the $t$-plane may be disregarded, for $t^{-\nu} J_{\nu}(t)$ is an even integral function of $t$. Since $\nu$ is here considered real and $\nu>-1$, the zeros of $t^{-\nu} J_{\nu}(t)=0$ are real, at $t= \pm \alpha_{s}(s=1,2,3)$. If $t^{-\sigma} F_{\sigma}(t)$ were another even integral function of $t$, where $\sigma>-1$, the ratio $t^{\nu-\sigma} F_{\sigma}(t) /\left(J_{\nu}\right)(t)$ is an even function of $t$. Suppose $f(t)$ is another analytic function that may possibly have simple zeros at $t=t_{n}$.

If

$$
\frac{t^{1+\nu-\sigma} F \sigma(t)}{f(t) J_{\nu}(t)} \rightarrow 0 \text { when } t \rightarrow \infty,
$$

the integral around a circle of radius $|t|=r$,

$$
\frac{1}{2 \pi i} \int \frac{t^{\nu-\sigma} F_{o}(t)}{f(t) J_{\nu_{0}}(t)} d t
$$

will approach zero when $r$ increases without limit and without passing through the points $t=t_{n}$ or $t= \pm \alpha_{s}$. By Cauchy's theorem this gives (since $J_{\nu}^{\prime}\left(\alpha_{s}\right)=-J_{\nu+1}\left(\alpha_{s}\right)$,

$$
\sum_{n} \frac{t_{n}^{\nu-\sigma} F_{\sigma}\left(t_{n}\right)}{f^{\prime}\left(t_{n}\right) J_{\nu}\left(t_{n}\right)}=\sum_{s=1}^{\infty} \frac{\alpha_{s}^{\nu-\sigma} F_{\sigma}\left(\alpha_{s}\right)}{J_{\nu+1}\left(\alpha_{s}\right)}\left[\frac{1}{f\left(\alpha_{s}\right)}-\frac{1}{f\left(-\alpha_{s}\right)}\right] .
$$

This would be a trivial identity $0=0$, only in the case where $f(t)$ is an even function of $t$.

Taking $f(t)=t-t_{0}$ gives the identity in $t$,

$$
t^{-\sigma} F_{\sigma}(t)=2 t^{-\nu} J_{\nu}(t) \sum_{s=1}^{\infty} \frac{\alpha_{s}^{1+\nu-\sigma} F_{\sigma}\left(\alpha_{s}\right)}{\left(\alpha_{s}^{2}-t^{2}\right) J_{\nu+1}\left(\alpha_{s}\right)},
$$

if

$$
\frac{t^{\nu-\sigma} \boldsymbol{F}_{\sigma}(t)}{J_{\nu}(t)} \rightarrow 0 \text { when } t \rightarrow \infty
$$

where $t^{-\sigma} F_{\sigma}(t)$ is an even integral function of $t$. Equation 9 applies when $\nu$ and $\sigma$ are both real and exceed -1 . Replacing $\sigma$ by $\nu+\sigma$ and taking $F_{\sigma+\nu}(t)=J_{\nu+\sigma}\left(t_{s}\right)$ gives

$$
J_{\nu+\sigma}(t \xi)=2 t^{\sigma} J_{\nu}(t) \sum_{s=1}^{\infty} \frac{\alpha_{s}^{1-\sigma} J_{\nu+\sigma}\left(\alpha_{s} \xi\right)}{\left(\alpha_{s}^{2}-t^{2}\right) J_{\nu+1}\left(\alpha_{s}\right)}
$$

for any $t$, when $0 \leqslant \xi<1$ if $-1<\nu$ and $-1<\nu+\sigma$ and when $\xi=1$ if $-1<\nu$ and $0<\bar{\sigma}$.

The case of this $(\xi=1, \sigma>0)$ is a special case $\mu=0$ of the following, which is obtained by use of the even function of $t$,

$$
t^{\nu} J_{\nu+\sigma}\left(\sqrt{t^{2}-\mu^{2}}\right) /\left[\left(t^{2}-\mu^{2}\right)^{\frac{\nu+\sigma}{2}} J_{\nu}(t)\right]
$$

$$
\begin{aligned}
& \frac{t^{\nu} J_{\nu+\sigma}\left(\sqrt{t^{2}-\mu^{2}}\right)}{\left(\sqrt{t^{2}-\mu^{2}}\right)^{\nu+\sigma}} \\
& \quad=2 J_{\nu}(t) \sum_{s=1}^{\infty} \frac{\alpha_{s}^{\nu+1} J_{\nu+\sigma}\left(\sqrt{\alpha_{s}^{2}-\mu^{2}}\right)}{\left(\alpha_{s}^{2}-t^{2}\right) J_{\nu+1}\left(\alpha_{s}\right)\left(\sqrt{\alpha_{s}^{2}-\mu^{2}}\right)^{\nu+\sigma}} .
\end{aligned}
$$

where $-1<\nu, 0<\sigma, t$ and $\mu$ arbitrary.

Another case of eq 9 with $F_{\sigma}(t)=G_{\nu}(t)$ :

If $t^{-\nu} G_{\nu}(t)$ is an even integral function of $t$ and $G_{\nu}(t) / J_{\nu}(t) \rightarrow 0$ when $t \rightarrow \infty\left(t \neq \alpha_{s}\right)$

then by eq. 9

$$
G_{\nu}(t)=2 J_{\nu}(t) \sum_{s=1}^{\infty} \frac{\alpha_{s} G_{\nu}\left(\alpha_{s}\right)}{\left(\alpha_{s}^{2}-t^{2}\right) J_{\nu+1}\left(\alpha_{s}\right)} .
$$

It will be found that the infinite set of functions,

$$
u_{s}(t) \equiv \frac{J_{\nu}(t)}{\alpha_{s}^{2}-t^{2}}(\text { where } s=1,2,3, \ldots \infty),
$$

constitute an open set of normal functions for the positive real interval $0<t<\infty$, That is

$$
\int_{0}^{\infty} t\left(\frac{J_{\nu}(t)}{\alpha_{s}^{2}-t^{2}}\right)\left(\frac{J_{\nu}(t)}{\alpha_{k}^{2}-t^{2}}\right) d t=\frac{1}{2 \alpha_{s}^{2}} \text { if } k=s,=0 \text { if } k \neq s .
$$

Since the set is not closed, an arbitrary function could not in general be developed in a series of these functions, but eq 10c shows that such developments are possible for a function $G_{\nu}(t)$ satisfying the conditions of eq 10a and $10 \mathrm{~b}$.

A function $G_{\nu}(t)$ would satisfy eq 10a if defined by a series

$$
G_{\nu}(t) \equiv 2 J_{\nu}(t) \sum_{s=1}^{\infty} \frac{x_{s}}{\alpha_{s}^{2}-t^{2}}=2 \sum_{s=1}^{\infty} x_{s} u_{s}(t),
$$

which would converge absolutely if $\left|x_{s}\right|<c s^{\mathrm{p}}$ for $s>s_{0}$ where $p<1$. Letting $t \rightarrow \alpha_{s}$ shows that

$$
x_{s}=\alpha_{s} G_{\nu}\left(\alpha_{s}\right) / J_{\nu+1}\left(\alpha_{s}\right) \text { as in }(10 \mathrm{c}) .
$$

To prove the set $u_{s}$ is not closed it is sufficient to find one function $f(t)$ (not a null-function) for which

$$
\int_{0}^{\infty} t f(t) u_{s}(t) d t=0 \text { for every } s(=1,2,3, \ldots \infty .
$$

A class of such functions will be found immediately; one of the simplest is $J_{\nu}(t \xi)$ where $1 \leqslant \xi$, for it will appear that

$$
\int_{0}^{\infty} t J_{\nu}(t \xi) u_{s}(t) d t=0 \text { for every } s .
$$

When eq 13 and 11b are proved, the following will be a consequence: 
If $G(t)$ is defined by a series (eq 12), or having the character of eq $10 \mathrm{a}$ and $10 \mathrm{~b}$ is developable in a series like eq 12 , then by eq 13

$$
\int_{0}^{\infty} t G_{\nu}(t) J_{\nu}(t \xi) d t=0 \text { when } 1 \leqslant \xi<\infty
$$

but when $0 \leqslant \xi<1$

$$
\int_{0}^{\infty} t G_{\nu}(t) J_{\nu}(t \xi) d t=g_{\nu}(\xi) \equiv 2 \sum_{s=1}^{\infty} \frac{G_{\nu}\left(\alpha_{s}\right) J_{\nu}\left(\alpha_{s} \xi\right)}{J_{\nu+1}^{2}\left(\alpha_{s}\right)} .
$$

These two equations show that $G_{\nu}(t)$ is the Hankel's transform of the function $g_{\nu}(\xi)$, which is zero for $\xi \geqslant 1$, where

$$
G_{\nu}(t)=\int_{0}^{1} \xi g_{\nu}(\xi) J_{\nu}(t \xi) d \xi .
$$

Conversely, if, with a given function $g_{\nu}(\xi)$, its transformation $G_{\nu}(\mathrm{t})$ were defined by this equation, the development (eq 14b) is the formula for developing $g_{\nu}(\xi)$ for $0 \leqslant \xi<1$ in the series of normal functions $J_{\nu}\left(\alpha_{s} \xi\right)$.

Equation 14a also follows from eq $14 \mathrm{c}$ by reason of Hankel's integral representation of $g_{\nu}(\xi)$. Finally the identity in $t$ of eq $10 \mathrm{c}$ would then be obtained by replacing $J_{\nu}(\xi t)$ in the integral (eq 14c) by the second member of eq 9a with $\sigma=0$.

The equivalence of eq $10 \mathrm{c}$ and $14 \mathrm{c}$ as to establishing eq 14a will be important to remember.

To derive eq $14 \mathrm{~b}$ for the case $0 \leqslant \xi<1$ we find by eq 9 a with $\sigma=0$

$$
\begin{gathered}
\int_{0}^{\infty} t G_{\nu}(t) J_{\nu}(\xi t) d t=2 \sum_{s=1}^{\infty} \frac{\alpha_{s} J_{\nu}\left(\alpha_{s} \xi\right)}{J_{\nu+1}\left(\alpha_{s}\right)} \int_{0}^{\infty} \frac{t J_{\nu}(t)}{\alpha_{s}^{2}-t^{2}} G_{\nu}(t) d t= \\
2 \sum_{s=1}^{\infty} \frac{\alpha_{s} J_{\nu}\left(\alpha_{s} \xi\right)}{J_{\nu+1}\left(\alpha_{s}\right)} \int_{0}^{\infty} t u_{s}(t) G_{\nu}(t) d t= \\
2 \sum_{s=1}^{\infty} \frac{\alpha_{s} J_{\nu}\left(\alpha_{s} \xi\right)}{J_{\nu+1}\left(\alpha_{s}\right)} \sum_{k=1}^{\infty} x_{k} 2 \int_{0}^{\infty} t u_{s}(t) u_{k}(t) d t . \quad \text { by eq }(12)
\end{gathered}
$$

The use of the orthogonal relations (eq 11b) leads to eq $14 \mathrm{~b}$.

We require next some transformations of the integral

$$
I=\int_{0}^{\infty} t G_{\nu}(t) J_{\nu}(\xi t) W(t) d t
$$

assumed to be convergent. The function $W(t)$ has no singularities in the immediate neighborhood of the real axis of $t$ and is an even function of $t$. $G_{\nu}(t)$ is defined by a series (eq 12), so that it has the same circuital relation around the branchpoint $t=0$ as $J_{\nu}(t)$, that is $G\left(t e^{ \pm i \pi}\right)=e^{ \pm i \nu_{\pi}} G_{\nu}(t)$.

As a first step displace the path of $I$ slightly downward so that it goes from $0-i 0$ to $\infty-i 0$ just below the positive real axis of $t$. After this replace $J_{\mu}(\xi t)$ by

$$
\frac{1}{2}\left[H_{\mu}(\xi t)+H_{\nu}^{(2)}(\xi t)\right] \text { eq. } 5 .
$$

Then

$$
\begin{aligned}
& I=\frac{1}{2} \int_{0-i 0}^{\infty-i 0} t G_{\nu}(t) H_{\nu}(\xi t) W(t) d t+ \\
& \frac{1}{2} \int_{0-i 0}^{\infty-i 0} t G_{\nu}(t) H_{\nu}^{(2)}(\xi t) W(t) d t .
\end{aligned}
$$

In the second integral let $t=t^{\prime} e^{-i \pi}$ and take account of the branch relations (eq 6). This gives

$$
I=\frac{1}{2} \int_{p} t G_{\nu}(t) H_{\nu}(\xi t) W(t) d t
$$

where the path $p$ begins at $t=-\infty+i \quad 0$ and continues above the cut (on negative real axis of $t$ ), crosses the axis at $t=+0$ and thence to $+\infty-$ $i 0$ below the positive real axis.

For the next transformation leave $J_{\nu}(\xi t)$ in the integrand of $I$ but break up $G_{\nu}(t)$. For this we may use temporarily the abbreviation $F(t)$ for the even function of $t$.

$$
F(t)=\sum_{s=1}^{\infty} \frac{x_{s}}{\alpha_{s}^{2}-t^{2}}
$$

so that eq 12 is $G_{\nu}(t)=2 F(t) J_{\nu}(t)=F(t)[H(t)+$ $\left.H^{(2)}(t)\right]$.

Hence

$$
\begin{aligned}
& I=\int_{0-i 0}^{\infty-i 0} t J_{\nu}(\xi t) F(t) W(t) H_{\nu}(t) d t+ \\
& \int_{0-i 0}^{\infty-i 0} t J_{\nu}(\xi t) F(t) W(t) H_{\nu}^{(2)}(t) d t .
\end{aligned}
$$

The substitution $t=t^{\prime} e^{-i \pi}$ in the second integral works out as before, giving

$$
I=\int_{p} t J_{\nu}(t \xi) F(t) W(t) H_{\nu}(t) d t .
$$

On restoring $G_{\nu}(t)$ by $F(t)=G_{\nu}(t) / 2 J(t)$ this gives

$$
\int_{0}^{\infty} G_{\nu}(t) J_{\nu}(\xi t) W(t) d t=\frac{1}{2} \int_{p} t G_{\nu}(t) H_{\nu}(t) \frac{J_{\nu}(\xi t)}{J_{\nu}(t)} W(t) d t
$$




$$
=\frac{1}{2} \int_{p} t G_{\nu}(t) H_{\nu}(\xi t) W(t) d t .
$$

where

$$
\int_{p} d t=\int_{-\infty+i 0}^{+0+i 0} d t+\int_{+0-i 0}^{+\infty-i 0} d t
$$

If the first integral converges, the only other assumption necessary for this transformation is that $t^{-\nu} G_{\nu}(t) W(t)$ be an even, analytic function of $t$ in a region that includes the real axis.

Taking $W(t)=1$ and $\mathrm{G}_{\nu}(t)=u_{s}(t)=J_{\nu}(t) /\left(\alpha_{s}^{2}-t^{2}\right)$ gives

$$
\begin{aligned}
\int_{0}^{\infty} t u_{s}(t) J_{\nu}(\xi t) d t & =\frac{1}{2} \int_{p} t u_{s}(t) H_{\nu}(\xi t) d t \\
& =\frac{1}{2 \pi i} \int_{p} \frac{i \pi t J_{\nu}(t) H_{\nu}(\xi t)}{\alpha_{s}^{2}-t^{2}} d t .
\end{aligned}
$$

On the infinite semicircle above the real axis

$$
\begin{aligned}
\left|i \pi t J_{\nu}(t) H_{\nu}(\xi t)\right| & \rightarrow 0 \text { if } 1<\xi \leqslant \infty \\
& \rightarrow 1 \text { if } \xi=1 .
\end{aligned}
$$

Hence, closing the path gives

$\int_{0}^{\infty} t u_{s}(t) J_{\nu}(\xi t) d t=0$ if $1 \leqslant \xi \leqslant \infty$, which is eq 13 .

Similarly,

$$
\begin{aligned}
\int_{0}^{\infty} t u_{s}(t) u_{k}(t) d t & =\int_{0}^{\infty} t\left[\frac{J_{\nu}(t)}{\alpha_{s}^{2}-t^{2}}\right] \cdot\left[\frac{J_{\nu}(t)}{\alpha_{k}^{2}-t^{2}}\right] d t \\
& =\frac{1}{2 \pi i} \int_{p} \frac{i \pi t H_{\nu}(t) J_{\nu}(t)}{\left(\alpha_{s}^{2}-t^{2}\right)\left(\alpha_{k}^{2}-t^{2}\right)} d t
\end{aligned}
$$

which gives the orthogonal relation (eq 11b).

The potential $V(x, \xi)$, which satisfies eq 1 , vanishes in the plane $x=0$ and has the value 1 on the right hand boundary of figure 1, may be formulated in terms of its (unknown) values on the artificial boundary $x=c, 0<\xi=\frac{\rho}{a}<1$.

Let

$$
\begin{aligned}
V(c, \xi) & =1-f_{\nu}(\xi)=1-\xi^{-v} g_{\nu}(\xi) \text { for } 0 \leqslant \xi \leqslant 1 \\
& =1 \text { for } 1 \leqslant \xi \leqslant \infty,
\end{aligned}
$$

where $f_{\nu}(1)=0=g_{\nu}(1)$.

Consider $g_{v}(\xi)$ the function represented by the series (eq 14b) and $G_{\nu}(t)$, its transform defined by eq $14 \mathrm{c}$ so that Hankel's integral identity is

$$
\begin{aligned}
\int_{0}^{\infty} t G_{\nu}(t) J_{\nu}(\xi t) d t & =g_{\nu}(\xi) \text { when } 0 \leqslant \xi \leqslant 1 \\
& =0 \text { when } 1 \leqslant \xi \leqslant \infty .
\end{aligned}
$$

Also the series (eq 10c) represents $G_{\nu}(t)$ for all values of $t$ and $t^{-\nu} G_{\nu}(t)$ is an even integral function of $t$. Since $g_{\nu}(\xi)$ vanishes when $\xi=1$, its representation in eq $14 \mathrm{~b}$ as a series of normal functions

$$
\phi_{s}(\xi)=\frac{\sqrt{2} J_{\nu}\left(\alpha_{s} \xi\right)}{J_{\nu+1}\left(\alpha_{s}\right)} \text { is valid for } 0 \leqslant \xi \leqslant 1 .
$$

The related set of normal functions,

$$
\psi_{s}(\xi)=\frac{\sqrt{2} J_{\nu+1}\left(\alpha_{s} \xi\right)}{J_{\nu+1}\left(\alpha_{s}\right)}
$$

are suitable for the development of the function

$$
\xi^{\nu} f_{\nu}{ }^{\prime}(\xi)=g_{\nu}{ }^{\prime}(\xi)-\frac{\nu}{\xi} g_{v}(\xi),
$$

for it is known by the general theory of these series of Dini's type that a function that becomes infinite when $\xi \rightarrow 1$ may be so developed provided its integral converges. The formula is

$$
\xi^{\nu} f_{\nu}^{\prime}(\xi)=2 \sum_{s=1}^{\infty} \frac{J_{\nu+1}\left(\alpha_{s} \xi\right)}{J_{\nu+1}^{2}\left(\alpha_{s}\right)} \int_{0}^{1} \xi_{1} J_{\nu+1}\left(\alpha_{s} \xi_{1}\right) \xi_{1}^{\nu} f_{\nu}^{\prime}\left(\xi_{1}\right) d \xi_{1} .
$$

That is, .

$$
\begin{aligned}
g_{\nu}^{\prime}(\xi)-\frac{\nu}{\xi} g_{\nu}(\xi)= \\
2 \sum_{s=1}^{\infty} \frac{J_{\nu+1}\left(\alpha_{s} \xi\right)}{J_{\nu+1}^{2}\left(\alpha_{s}\right)} \int_{0}^{1} \xi_{1} J_{\nu+1}\left(\alpha_{s} \xi_{1}\right)\left[g_{\nu}^{\prime}\left(\xi_{1}\right)-\frac{\nu}{\xi_{1}} g_{\nu}\left(\xi_{1}\right)\right] d \xi_{1} .
\end{aligned}
$$

By integration by parts the integral is found to be

$$
-\int_{0}^{\prime}\left[\alpha_{s} \xi_{1} J_{\nu+1}^{\prime}\left(\alpha_{s} \xi_{1}\right)+(\nu+1) J_{\nu+1}\left(\alpha_{s} \xi_{1}\right)\right] g_{\nu}\left(\xi_{1}\right) d \xi_{1} .
$$

By use of eq 3 and $3 \mathrm{a}$ this reduces to

$$
-\alpha_{s} \int_{0}^{\prime} \xi_{1} g_{\nu}\left(\xi_{1}\right) J_{\nu}\left(\alpha_{s} \xi_{1}\right) d \xi_{1}
$$

which is $-\alpha_{s} G_{\nu}\left(\alpha_{s}\right)$, so that

$$
\begin{aligned}
& \xi_{\nu} \frac{d}{d \xi}\left[\xi^{-\nu} g_{\nu}(\xi)\right]=g_{\nu}^{\prime}(\xi)- \\
& \frac{\nu}{\xi} g_{\nu}(\xi)=-2 \sum_{s=1}^{\infty} \frac{\alpha_{s} G_{\nu}\left(\alpha_{s}\right)}{J_{\nu+1}^{2}\left(\alpha_{s}\right)} J_{\nu+1}\left(\alpha_{s} \xi\right),
\end{aligned}
$$

which is valid for $0 \leqslant \xi<1$. As $\xi \rightarrow 1$ this series converges to values that increases without limit, 
as it must do to represent the function on the left in which $f_{\nu}^{\prime}(\xi)$ and $g_{\nu}^{\prime}(\xi)$ go to $-\infty$. This method of derivation of eq 18 proves that it is permissible to make term-by-term application to eq 14a of the differentiating operator $\xi^{\nu} \frac{d}{d \xi}\left[\xi^{-\nu} g_{\nu} \xi\right]$, for this gives eq 18 .

To test the convergence of series and integrals representing the potential, and to make certain transformation of these integrals, a knowledge of the principal term in the asymptotic expansion of $G_{\nu}(t)$ is required. These may be derived from the well-known fact that if the interior angle at $A_{2}$ in figure 1 is $\theta$, the potential in its neighborhood must vanish like $A\left(1-\xi^{2}\right)^{\pi / \theta}$. Since $\theta$ is $3 \pi / 2$ in this problem, consider the comparisonfuntion

$$
\bar{g}_{\nu}(\xi)=\xi^{\nu} \bar{f}_{\nu}(\xi)=A_{\nu} \xi^{\nu}\left(1-\xi^{2}\right)^{2 / 3} \text { where } A_{\nu}>0 .
$$

Its transform is

$$
\bar{G}_{\nu}(t)=A_{\nu} \int_{0}^{1} \xi^{\nu+1}\left(1-\xi^{2}\right)^{2 / 3} J_{\nu}(t \xi) d \xi .
$$

Hence $\bar{g}_{\nu}(\xi)$ is represented by a series like eq $14 \mathrm{~b}$ and also by eq 18

$$
\bar{g}_{\nu}^{\prime}(\xi)-\frac{\nu}{\xi} \bar{g}_{\nu}(\xi)=-2 \sum_{s=1}^{\infty} \frac{\alpha_{s} \bar{G}_{\nu}\left(\alpha_{s}\right)}{J_{\nu+1}^{2}\left(\alpha_{s}\right)} J_{\nu+1}\left(\alpha_{s} \xi\right)
$$

In the actual potential in both cases $\nu=0$ and $\nu=$ $-1 / 2$ the potential must vanish at $A_{2}$ so that $g_{v}$ vanishes like the comparison-function $\bar{g}_{\nu}(\xi)$ when $\xi \rightarrow 1$. Consequently there must be a finite limit $L_{\nu}$ such that when $\xi \rightarrow 1$

$$
g_{\nu}(\xi)-\bar{g}_{\nu}(\xi)=\infty-\infty=L_{\nu} .
$$

This finite constant $L_{\nu}$ may be positive, negative, or *zero. Its value is not required, nor is that of the positive constant $A$.

If we subtract from eq $10 \mathrm{c}$ the corresponding identity,

$$
\bar{G}_{\nu}(t)=2 J_{\nu}(t) \sum_{s=1}^{\infty} \frac{\alpha_{s} \bar{G}_{\nu}\left(\alpha_{s}\right)}{\left(\alpha_{s}^{2}-t^{2}\right) J_{\nu+1}^{\left(\alpha_{s}\right)}}
$$

we get, after multiplying the resulting 'equation by $t^{2} / J_{\nu}(t)$,

$$
\frac{t^{2}\left[G_{\nu}(t)-\bar{G}_{\nu}(t)\right]}{J_{\nu}(t)}=-2 \sum_{s=1}^{\infty} \frac{\alpha_{s}}{J_{\nu+1}^{\left(\alpha_{s}\right)}}\left[\frac{G_{\nu}\left(\alpha_{s}\right)-\bar{G}_{\nu}\left(\alpha_{s}\right)}{1-\frac{\alpha_{s}^{2}}{t^{2}}}\right] .
$$

Subtracting eq 19a from eq 18 we get in the limit (since $\bar{g}_{\nu}(\xi) \rightarrow 0$ and $g_{\nu}(\xi) \rightarrow 0$ when $\xi \rightarrow 1$ )

\section{limit}

$$
\begin{aligned}
& \operatorname{limit}_{\xi \rightarrow 1\left[g_{\nu}^{\prime}(\xi)-g_{\nu}^{\prime}(\xi)\right]}=\infty-\infty=L_{\nu} \\
&=-2 \sum_{s+1}^{\infty} \frac{\alpha_{s}}{J_{\nu+1}\left(\alpha_{s}\right)}\left[G_{\nu}\left(\alpha_{s}\right)-\bar{G}_{\nu}\left(\alpha_{s}\right)\right],
\end{aligned}
$$

which shows that the second member of the preceding equation approaches some of finite limit $L_{\nu}$ when $t \rightarrow \infty$ without passing through any zero $\alpha_{s}$ of $J_{\nu}(t)$.

Consequently the principal term in the asymptotic expansion of $G_{\nu}(t)$ is contained in

$$
G_{\nu}(t) \sim \bar{G}_{\nu}(t)+\frac{L_{\nu}}{t^{2}} J_{\nu}(t) .
$$

To see which of these two terms is the important one, we may find $\bar{G}_{\nu}(t)$ by application of Sonin's integral. If $\sigma$ and $\nu$ are real fand $\sigma>0$, $\nu>-1$, while $t$ and $\mu$ are arbitrary, Sonin's integral is

$$
\begin{aligned}
& (i \mu)^{1-\sigma} \int_{0}^{\pi / 2} \cos ^{\nu+1} \phi \sin { }^{\sigma} \phi J_{\sigma-1}(i \mu \sin \phi) J_{\nu}(t \cos \phi) d \phi= \\
& (i \mu)^{1-\sigma} \int_{0}^{1} \xi J_{\nu}(\xi t)\left[\xi^{\nu}\left(1-\xi^{2}\right)^{\frac{\sigma-1}{2}} J_{\sigma-1}\left(i \mu \sqrt{1-\xi^{2}}\right)\right] d \xi= \\
& \frac{t^{\nu} J_{\nu+\sigma}\left(\sqrt{\left.t^{2}-\mu^{2}\right)}\right.}{\left(\sqrt{t^{2}-\mu^{2}}\right)^{\nu+\sigma}}
\end{aligned}
$$

which becomes with $\mu=0$

$$
\int_{0}^{1} \xi^{\nu+1}\left(1-\xi^{2}\right)^{\sigma-1} J_{\nu}(\xi t) d \xi=\frac{\Gamma(\sigma)}{2}\left(\frac{2}{t}\right)^{\sigma} J_{\nu+\sigma}(t) .
$$

Hence taking $\sigma=5 / 3$, eq $19 \mathrm{~b}$ gives

$$
\bar{G}_{\nu}(t)=\frac{A_{\nu}}{2}\left(\frac{2}{t}\right)^{5 / 3} J_{\nu+5 / 3}(t) .
$$

When $t$ is large this is the important term in eq 20 so the asymptotic expression for $G_{\nu}(t)$ may be taken in the form

$$
t^{5 / 3} \frac{G_{\nu}(t)}{J_{\nu}(t)} \sim \text { constant when } t \rightarrow \infty\left(t \neq \alpha_{s}\right) .
$$

If the interior angle at $A_{2}$ of figure 1 were any other than $\theta=3 / 2$, say $\pi<\theta<2 \pi$ the factor $t^{5 / 3}$ would be replaced by $t^{1+\pi / \theta}$.

The formulation of the potential will be implicitly in terms of $g_{\nu}(\xi)$ but explicitly in terms of its transforma $G_{\nu}(t)$. 
All that is assumed as to $G_{\nu}(t)$ is that $t^{-\nu} G_{\nu}(t)$ is an even integral function of $t$ with asymptotic expansion (eq 23).

If $\gamma=c / a$ and $\beta=(b-c) / a$, the potential in region I may be written as an integral over a path $p_{1}$, which encircles in positive direction the positive real axis of $t$, (all the positive zeros $t=\alpha_{s}$ of $\left.J_{\nu}(t)=0\right)$.

In region $\mathrm{I}$

$$
V(x, \xi)=1-\frac{\xi^{-\nu}}{2} \int_{p_{1}} t G_{\nu}(t) H_{\nu}(t) \frac{J_{\nu}(t \xi) \sinh (b-x) t / a}{J_{\nu}(t) \sinh \beta t} d t
$$

In the combined region II and III

$$
V(x, \xi)=\frac{x}{c}-\frac{\xi^{-\nu}}{2} \int_{p} t G_{\nu}(t) H_{\nu}(t) \frac{J_{\nu}(t \xi) \sinh x t / a}{J_{\nu}(t) \sinh \gamma t} d t .
$$

To show that this potential is everywhere continuous and satisfies the required boundary conditions, consider the first integral in eq 24a. This vanishes when $x=b$ and again when $\rho=a$, $(\xi=1)$ since the denominator $J_{\nu}(t)$ disappears, and the path $p_{1}$ then encloses no singular points. At the internal boundary $x=c$ the integrands in eq 24a and $24 \mathrm{~b}$ become equal. Also, since the denominators $\sinh \beta t$ and $\sinh \gamma t$ disappear the integrands have no singularity above the real axis of $t$ so the paths $p$ and $p_{1}$ become equivalent. Consequently the potential is continuous at $x=c, 0 \leqslant \xi \leqslant 1$.

The integral in eq $24 \mathrm{~b}$ and the potential vanish when $x=0$. To see that the integral (eq 24b) also vanishes when $\xi \rightarrow \infty$, reference to eq 15 shows that eq $24 \mathrm{~b}$ is equivalent to two other integrals all equally valid in the combined region II and III.

$$
V(x, \xi)=\frac{x}{c}-\frac{\xi^{-\nu}}{2} \int_{p} t G_{\nu}(t) H_{\nu}(t \xi) \frac{\sinh x t / a}{\sinh \gamma t} d t
$$

and

$V(x, \xi)=\frac{x}{c}-\xi^{-\nu} \int_{0}^{\infty} t G_{\nu}(t) J_{\nu}(t \xi) \frac{\sinh x t / a}{\sinh \gamma t} d t$.

By eq 23 the path $p$ of eq 24c may be closed with an infinite semicircle above the real axis of $t$, when $\xi \geqslant 1$. It encircles the poles at $t=i n \pi / \gamma$ where $\sinh \gamma t=0$, so that

in region III $(0 \leqslant x \leqslant c$ and $a \leqslant \rho \leqslant \infty)$

$$
V(x, \xi)=\frac{x}{c}+\frac{\pi \xi^{-\nu}}{\gamma} \sum_{n=1}^{\infty}(-1)^{n} \sin \left(\frac{n \pi x}{c}\right)\left(\frac{i n \pi}{\gamma}\right)
$$

$$
H_{\nu}\left(\frac{i n \pi}{\gamma} \xi\right) G_{\nu}\left(\frac{i n \pi}{\gamma}\right)
$$

In region II where $0 \leqslant \xi<1$ it is eq $24 \mathrm{~b}$ whose path may be closed with the same semicircle. The path then encircles two sets of poles, the set where $\sinh \gamma t=0$ and the set $t=\alpha_{s}$ on the positive real axis of $t$. Hence in region II (where $0 \leqslant x \leqslant c$ and $0 \leqslant \rho \leqslant a$ ) eq $24 \mathrm{~b}$ is equivalent to the series

$$
\begin{aligned}
V(x, \xi)= & \frac{x}{c}-2 \xi^{-\nu} \sum_{s=1}^{\infty} \frac{G_{\nu}\left(\alpha_{s}\right) J_{\nu}\left(\alpha_{s} \xi\right) \sinh x \alpha_{s} / a}{J_{\nu+1}^{2}\left(\alpha_{s}\right) \sinh \gamma \alpha_{s}}+ \\
& \frac{\pi \xi^{-\nu}}{\gamma} \sum_{n=1}^{\infty}(-1)^{n} \sin \left(\frac{n \pi x}{c}\right)\left(\frac{i n \pi}{\gamma}\right) H_{\nu} \\
& \left(\frac{i n \pi}{\gamma}\right) G_{\nu}\left(\frac{i n \pi}{\gamma}\right) \frac{J_{\nu}(i n \pi \xi / \gamma)}{J_{\nu}(i n \pi / \gamma)} .
\end{aligned}
$$

Similarly in region I (where $c \leqslant x \leqslant b$ and $0 \leqslant \rho \leqslant a$ ) eq 24 a is equivalent to

$V(x, \xi)=1-2 \xi^{-\nu} \sum_{s=1}^{\infty} \frac{G \nu\left(\alpha_{s}\right) J_{\nu}\left(\alpha_{s} \xi\right) \sinh (b-x) \alpha_{s} / a}{J^{2}{ }_{\nu+1}\left(\alpha_{s}\right) \sinh \beta \alpha_{s}}$.

From the last two series, we get the series that states the continuity of $D_{x} V$,

$c \xi^{\nu}\left[\left(D_{x} V\right)_{x=c+0}-\left(D_{x} V\right)_{x=c-0}\right]=$

$2 \sum_{s=1}^{\infty} \frac{J_{\nu}\left(\alpha_{s} \xi\right)}{\alpha_{s} J_{\nu+1}\left(\alpha_{s}\right)}\left[\gamma \alpha_{s}\left(\operatorname{coth} \gamma \alpha_{s}+\operatorname{coth} \beta \alpha_{s}\right) \frac{\alpha_{s} G_{\nu}\left(\alpha_{s}\right)}{J_{\nu+1}\left(\alpha_{s}\right)}\right]-$

$\pi \sum_{n=1}^{\infty}\left(\frac{n \pi}{\gamma}\right) \frac{J_{\nu}(i n \pi \xi / \gamma)}{J_{\nu}(i n \pi / \gamma}\left(\frac{i n \pi}{\gamma}\right) H_{\nu}\left(\frac{i n \pi}{\gamma}\right) G_{\nu}\left(\frac{i n \pi}{\gamma}\right)-$

$\xi^{\nu}=0$,

which must be true only for $0 \leqslant \xi<1$.

For this interval

$$
\xi^{\nu}=2 \sum_{s=1}^{\infty} \frac{J_{\nu}\left(\alpha_{s} \xi\right)}{\alpha_{s} J_{\nu+1}\left(\alpha_{s}\right)} .
$$

Also by eq 9 a with $\sigma=0$ and $t=i n \pi / \nu$

$$
\frac{J_{\nu}(i n \pi \xi / \gamma)}{J_{\nu}(i n \pi / \gamma)}=2 \sum_{s=1}^{\infty} \frac{J_{\nu}\left(\alpha_{s} \xi\right)}{\alpha_{s} J_{\nu+1}\left(\alpha_{s}\right)}\left[\frac{\alpha_{s}^{2}}{\alpha_{s}^{2}+(n \pi / \gamma)^{2}}\right]
$$

Placing these in eq 27 gives a series $\sum_{1}^{\infty} C_{s} J_{0}\left(\alpha_{s} \xi\right)=0$ so each coefficient $C_{s}$ must vanish.

Hence the continuity of $D_{x} V$ at $x=c$ requires that $\mathrm{G}_{\nu}(t)$ be such a function as to satisfy the infinite system of linear equations, in which, for brevity,

$$
A_{s} \equiv \frac{1}{\operatorname{coth} \gamma \alpha_{s}+\operatorname{coth} \beta \alpha_{s}}=\frac{\sinh \gamma \alpha_{s} \sinh \beta \alpha_{s}}{\sinh (\beta+\gamma) \alpha_{s}}
$$


and

$$
x_{s} \equiv \frac{\alpha_{s} G_{\nu}\left(\alpha_{s}\right)}{J_{\nu+1}\left(\alpha_{\tau}\right)} .
$$

The system of equations $(s=1,2,3, \ldots \infty)$ for continuity of $D_{x} V$ is

$x_{s}-A_{s} \alpha_{s} \sum_{n=1}^{\infty} \frac{n \pi\left[\pi(i n \pi / \gamma) H_{\nu}(i n \pi / \gamma)\right]}{(n \pi)^{2}+\left(\gamma \alpha_{s}\right)^{2}} G_{\nu}\left(\frac{i n \pi}{\gamma}\right)=\frac{A_{s}}{\gamma \alpha_{s}}$.

The additional condition, that $V(c, \xi)=1$ for $\xi>1$ is the equivalent of the identity in $t$ in eq $10 \mathrm{c}$, which becomes

$$
G\left(\frac{i n \pi}{\gamma}\right)=2 \gamma^{2} J_{\nu}\left(\frac{i n \pi}{\gamma}\right) \sum_{k=1}^{\infty} \frac{x_{k}}{(n \pi)^{2}+\left(\gamma \alpha_{k}\right)^{2}} .
$$

There is an infinite class of functions $G_{\nu}(t)$ that satisfy eq 32a making $D_{x} V$ continuous. There is another infinite class satisfying the identity (eq 10c) (or eq $32 \mathrm{~b}$ in particular). These make $V(c, \xi)=1$ when $\rho>a$. The function common to both classes will be uniquely determined. The second condition (eq $10 \mathrm{c}$ or $32 \mathrm{~b}$ ) could be replaced by the statement that $G_{\nu}(t)$ is that solution of eq $32 \mathrm{a}$ such that $t^{-\nu} G_{\nu}(t)$ is an even integral function of $t$ with asymptotic expansion (eq 23). For this property is all that was required to show that the potential of eq 24a and $24 \mathrm{~b}$ satisfies all the external boundary conditions and is continuous at $x=c$ for $0 \leqslant \xi \leqslant 1$. Hence if eq $32 \mathrm{~b}$ is true, the potential is thereby determined and $D_{x} V$ is continuous. This potential is known to be unique.

Writing for brevity

$X_{s k}^{(1)}(\gamma)=X_{k s}^{(1)}(\gamma) \equiv \sum_{n=1}^{\infty} \frac{2 n \pi\left[\pi(i n \pi / \gamma) H_{\nu}(i n \pi / \gamma) J_{\nu}(i n \pi / \gamma)\right]}{\left[(n \pi)^{2}+\left(\gamma \alpha_{s}\right)^{2}\right]\left[(n \pi)^{2}+\left(\gamma \alpha_{k}\right)^{2}\right]}$,

the result of eliminating $G_{\nu}(i n \pi / \gamma)$ between eq $32 \mathrm{a}$ and $32 \mathrm{~b}$ is

$$
x_{s}-\gamma^{2} A_{s} \alpha_{s} \sum_{k=1}^{\infty} X_{r k}^{(1)} x_{\kappa}=\frac{A_{s}}{\gamma \alpha_{s}},
$$

which was derived more briefly in the text.

If the $s^{t h}$ eq 34 is multiplied by $2 J_{\nu}\left(\alpha_{s} \xi\right) /$ $\alpha_{s} J_{p+1}\left(\alpha_{s}\right)$ and summed, this gives, by reference to eq $14 \mathrm{~b}$ and 31

$$
\begin{aligned}
& g_{\nu}(\xi)-\gamma^{2} \sum_{k=1}^{\infty} \sum_{s=1}^{\infty} 2 A_{s} \alpha_{k} X_{s k}^{(1)}\left[\frac{J_{\nu}\left(\alpha_{s} \xi\right)}{J_{\nu+1}\left(\alpha_{s}\right)}\right] \cdot \frac{G_{\nu}\left(\alpha_{k}\right)}{J_{\nu+1}\left(\alpha_{k}\right)}= \\
& \frac{2}{\gamma_{s=1}^{\infty}} \frac{A_{s} J_{\nu}\left(\alpha_{s} \xi\right)}{\alpha_{s}^{2} J_{\nu+1}\left(\alpha_{s}\right)} .
\end{aligned}
$$

Since $G_{\nu}\left(\alpha_{k}\right)=\int_{0}^{1} \xi_{1} g_{\nu}\left(\xi_{1}\right) J_{\nu}\left(\alpha_{k} \xi_{1}\right) d \xi_{1}$ this is an integral equation to determine $g_{\nu}(\xi)$ for $0 \leqslant \xi \leqslant 1$.

$$
g_{\nu}(\xi)-\gamma^{2} \int_{0}^{1} \xi_{1} g_{\nu}\left(\xi_{1}\right) N\left(\xi, \xi_{1}\right) d \xi_{1}=\frac{2}{\gamma} \sum_{s=1}^{\infty} \frac{A_{s} J_{\nu}\left(\alpha_{s} \xi\right)}{\alpha_{s}^{2} J_{\nu+1}\left(\alpha_{s}\right)}
$$

where

$$
\left.N\left(\xi, \xi_{1}\right)=2 \sum_{s=1}^{\infty} \sum_{k=1}^{\infty} A_{s} \alpha_{k} X_{s k}^{(\prime)} \frac{J_{\nu}\left(\alpha_{s} \xi\right) J_{\nu}\left(\alpha_{k} \xi_{1}\right)}{J_{\nu+1}\left(\alpha_{s}\right) J_{\nu+1}\left(\alpha_{k}\right)} \cdot\right\}
$$

It is known that the solution of this integral equation of Fredholm's type is given by the method of Neumann-Liouville, in a convergent series of iterated integrals, provided that $\gamma^{2}$ is less than $\left|\lambda_{1}\right|$ where $\lambda_{1}$ is the smallest characteristic constant, or root of the Fredholm determinant formed from $N$. The constants will of course be functions of $\beta$ and $\gamma$. This suggested testing the Neumann-Liouville method, which in effect is the method of successive substitution that was proved in the text to give the solution of the system (eq 34) for all positive values of $\beta$ and $\gamma$. Hence it may be concluded that $\lambda_{1}$ is a function of $\beta$ and $\gamma$ such that $\left|\lambda_{1}\right|$ is always greater than $\gamma^{2}$ (unless $N$ is a kind of nucleus with no eigen-constants). It may be shown that this is not the case, and that $N$ has an infinite number of constants $\lambda_{s}$ all real.

For computation the system (eq 34) is disappointing, and, although it admits of a great variety of interesting transformations, these will probably all be unsatisfactory until someone discovers the appropriate normal functions for $N$ or what amounts to an orthogonal transformation reducing the double series in eq 35 to diagonal terms only.

In view of the fact that the potential problem for the two-dimensional potential $(\nu=-1 / 2)$ admits an exact (though implicit) solution by conformal mapping with theta-functions it does not seem improbable that someone might solve the problem here formulated for general values of $\nu$ or at least for $\nu=0$.

With this in view we add a few remarks and other integral equations, which so far have led nowhere.

In the series of eq 34 we may place

$$
x_{k} \equiv \frac{\alpha_{k} G_{\nu}\left(\alpha_{k}\right)}{J_{\nu+1}\left(\alpha_{k}\right)}=\alpha_{k}^{2} \int_{0}^{\infty} \frac{t_{1} J_{\nu}\left(t_{1}\right) G_{\nu}\left(t_{1}\right)}{\alpha_{s}^{2}-t_{1}^{2}} d t_{1}
$$




$$
=\frac{\alpha_{k}^{2}}{2} \int_{p} \frac{t_{1} G_{\nu}\left(t_{1}\right) H_{\nu}\left(t_{1}\right)}{\alpha_{k}^{2}-t_{1}^{2}} d t_{1}
$$

Hence if we multiply the $s^{\text {th }}$ equation of the system (eq 34) by $2 J_{\nu}(t) /\left(\alpha_{s}-t^{2}\right)$ and $\operatorname{sum} s=1$ to $\infty$ this gives

$$
\begin{gathered}
G_{\nu}(t)-2 \gamma^{2} \sum_{s=1}^{\infty} \sum_{k=1}^{\infty} \frac{A_{s} \alpha_{s} X_{s k}^{(1)} x_{k} J_{\nu}(t)}{\alpha_{s}^{2}-t^{2}}= \\
\frac{2 J_{\nu}(t)}{\gamma} \sum_{s=1}^{\infty} \frac{A_{s}}{\alpha_{s}\left(\alpha_{s}^{2}-t^{2}\right)}
\end{gathered}
$$

Replacing $x_{k}$ by the first integral above gives

$\left.G_{\nu}(t)-\gamma^{2} \int_{0}^{\infty} t_{1} G_{\nu}\left(t_{1}\right) M\left(t, t_{1}\right) d t_{1}=\frac{2 J_{\nu}(t)}{\gamma} \sum_{s=1}^{\infty} \frac{A_{s}}{\alpha_{s}\left(\alpha_{s}^{2}-t^{2}\right)}\right)$

where

$M\left(t, t_{1}\right)=2 \sum_{s=1}^{\infty} \sum_{k=1}^{\infty} A_{s} \alpha_{k} X_{s k}^{(11}\left[\frac{\alpha_{s} J_{\nu}(t)}{\alpha_{s}^{2}-t^{2}}\right] \cdot\left[\frac{\alpha_{k} J_{\nu}(t)}{\alpha_{k}^{2}-t^{2}}\right]$.

This integral equation satisfied by $G_{\nu}(t)$ for $0<t \infty$ is formally similar to its transform, the integral equation eq 35 , which determines $g_{\nu}(\xi)$ for $0<\xi<1$. The nucleus $M\left(t, t_{1}\right)$ is formed from $N \quad\left(\xi, \quad \xi_{1}\right)$ by replacing the normal functions $J_{\nu}\left(\alpha_{s} \xi\right) / J_{\nu+1}\left(\alpha_{s}\right)$ in the double series by the normal function $\left.\alpha_{s} J_{\nu}(t) / \alpha_{s}^{2}-t^{2}\right)$.

This set of normal functions is not closed, but this is compensated by the fact that the subsidiary relation (eq 10c) requires that the solution $G_{\nu}(t)$ be a function that is developable in terms of them.

The series in eq 32a may be put in the form of an integral, for

$\frac{1}{2} \int_{p} t_{1}^{2} \operatorname{coth} \gamma t_{1} H_{\nu}\left(t_{1}\right) G_{\nu}\left(t_{1}\right) \frac{d t_{1}}{\alpha_{s}^{2}-t_{1}^{2}}=\frac{1}{2} x_{s} \operatorname{coth} \gamma \alpha_{s}-$

$$
\sum_{n=1}^{\infty} \frac{n \pi\left[\pi(i n \pi / \gamma) H_{\nu}(i n \pi / \gamma)\right]}{(n \pi)^{2}+\left(\gamma \alpha_{s}\right)^{2}} G_{\nu}\left(\frac{i n \pi}{\gamma}\right) .
$$

Hence the system of eq $32 \mathrm{a}$ is equivalent to

$$
\begin{aligned}
& \gamma x_{s}+\frac{\alpha_{s} \tanh \beta \alpha_{s}}{2} \int_{p} t_{1} G_{\nu}\left(t_{1}\right)\left(\gamma t_{1}\right) \operatorname{coth}\left(\gamma t_{1}\right) H_{\nu}\left(t_{1}\right) \\
& \frac{d t_{1}}{\alpha_{s}^{2}-t_{1}^{2}}=\frac{\tanh \beta \alpha_{s}}{\alpha_{s}} .
\end{aligned}
$$

Multiplying by $2 J_{\nu}(t) /\left(\alpha_{s}^{2}-t^{2}\right)$ and summing gives $\gamma G_{\nu}(t)+\frac{J_{\nu}(t)}{2} \int_{p} t_{1} G_{\nu}\left(t_{1}\right) \frac{\gamma t_{1} H_{\nu}\left(t_{1}\right)}{\tanh t_{1} \gamma} \sum_{s=1}^{\infty} \frac{2 \alpha_{s} \tanh \beta \alpha_{s}}{\left(\alpha^{2}-t^{2}\right)\left(\alpha_{s}^{2}-t_{1}^{2}\right)}$ $d t_{1}=2 J_{\nu}(t) \sum_{s=1}^{\infty} \frac{\tanh \beta \alpha_{s}}{\alpha_{s}\left(\alpha_{s}^{2}-t^{2}\right)}$,

which may also be put in the form

$\gamma G_{\nu}(t)+\int_{0}^{\infty} t_{1} G_{\nu}\left(t_{1}\right) K\left(t, t_{1}\right) d t_{1}=2 J_{\nu}(t) \sum_{s=1}^{\infty} \frac{\tanh \beta \alpha_{s}}{\alpha_{s}\left(\alpha_{s}^{2}-t^{2}\right)}$,

where

$$
\begin{aligned}
& K\left(t, t_{1}\right)= \\
& \gamma t_{1} \operatorname{coth} \gamma t_{1} \sum_{s=1}^{\infty} 2 \alpha_{s} \tanh \beta \alpha_{s}\left[\frac{J_{\nu}(t)}{\alpha_{s}^{2}-t^{2}}\right]\left[\frac{J_{\nu}\left(t_{1}\right)}{\alpha_{s}^{2}-t_{1}^{2}}\right]
\end{aligned}
$$

One method of avoiding the system (eq 34) consists in assuming such an expansion in series for $G_{\nu}(t)$ that the eq $10 \mathrm{c}$ becomes an identity in $t$ without placing any reductions upon the constants or coefficients of the assumed series. In that case the set of eq $32 \mathrm{a}$ would be the only equations to be satisfied.

Such a form would be obtained for $G_{\nu}(t)$ if it is the Hankel's transformation of any suitable function $g_{v}(\xi)$ as in eq $14 \mathrm{c}$.

Sonin's integral suggests a form for $g_{\nu}(\epsilon)$ that contains an arbitrary constant $\mu$ and arbitrary parameters $\sigma$.

$$
\begin{aligned}
& \text { If } \\
& g_{\nu}(\xi)=(i \mu)^{1-\sigma} \sum_{m} B_{m} \xi^{\nu}\left(1-\xi^{2}\right)^{\frac{\sigma_{m}-1}{2}} J_{\sigma_{m}-1}\left(i \mu \sqrt{1-\xi^{2}}\right)
\end{aligned}
$$

where $\sigma_{m} \geqslant 5 / 3$

Then Sonin's integral (eq 21) gives

$$
G_{\nu}(t)=t^{\nu} \sum_{m} B_{m} \frac{J_{\nu+\sigma}\left(\sqrt{t^{2}-\mu^{2}}\right)}{\left(\sqrt{t^{2}-\mu^{2}}\right)^{\nu+\sigma_{m}}} .
$$

With this expression eq 10c would be an identity in $t$ without placing restrictions upon the constants $B_{m}$ (other than required for convergence). This may be verified directly by use of eq $9 \mathrm{~b}$ in eq $14 \mathrm{c}$.

If $G_{\nu}(t)$ is defined by a convergent series (eq 39b), then $t^{-\nu} G_{\nu}(t)$ is an even integral function of $t$. If the smallest value of $\sigma_{m}$ is $5 / 3$, then $G_{\nu}(t)$ has the asymptotic expansion of eq 23.

The set of equations (eq 32a) become the following set to determine $B_{m}$

$$
\sum_{m} B_{m}\left\{\frac{\alpha_{s}^{\nu} J_{\nu+\sigma}\left(\sqrt{\alpha_{s}^{2}-\mu^{2}}\right)}{A_{s} J_{\nu+1}\left(\alpha_{s}\right)\left(\sqrt{\alpha_{s}^{2}-\mu^{2}}\right)^{\nu+\sigma_{m}}}-\pi \sum_{n=1}^{\infty}\right.
$$


$\left.\frac{n \pi(i n \pi / \gamma)^{\nu+1} H_{\nu}(i n \pi / \gamma) J_{\nu+\sigma}\left(i \sqrt{\left(\frac{n \pi}{\gamma}\right)^{2}+\mu^{2}}\right)}{\left[(n \pi)^{2}+\left(\gamma \alpha_{s}\right)^{2}\right]\left(i \sqrt{\left.\left(\frac{n \pi}{\gamma}\right)^{2}+\mu^{2}\right)^{\nu+\sigma_{m}}}\right.}\right\}=\frac{1}{\gamma \alpha_{s}^{2}}$

$s=1,2,3 \ldots$

Taking $\mu=0$ and $\sigma_{m}=m / 3 \quad(m=5,6,7, \ldots$

$$
\begin{aligned}
& g_{\nu}(\xi)=2 \xi^{\nu} \sum_{m=5}^{\infty} \frac{B_{m}\left(1-\xi^{2}\right)^{\frac{m}{3}-1}}{2^{m / 3} \Gamma(m / 3)} \\
& G_{\nu}(t)=\sum_{m=5}^{\infty} B_{m} t^{-m / 3} J_{\nu+m / 3}(t)
\end{aligned}
$$

$$
\begin{gathered}
\sum_{m=5}^{\infty} B_{m}\left\{\frac{J_{\nu+m / 3}\left(\alpha_{s}\right)}{A_{s} \alpha_{s}^{m / 3} J_{\nu+1}\left(\alpha_{s}\right)}-\right. \\
\left.\pi \sum_{n=1}^{\infty} \frac{n \pi H_{\nu}\left(\frac{i n \pi}{\gamma}\right) J_{\nu+m / 3}(i n \pi / \gamma)}{\left(\frac{i n \pi}{\gamma}\right)^{\frac{m}{3}-1}\left[(n \pi)^{2}+\left(\gamma \alpha_{s}\right)^{2}\right]}\right\}=\frac{1}{\gamma \alpha_{s}^{2}} .
\end{gathered}
$$

which might require less labor in computing $B_{m}$ than is required to compute $x_{s}$ from eq 34 .

WASHINGTON, January 23, 1948. 